\title{
Analytical Models for Hurricanes
}

\author{
Arkadii I. Leonov \\ Departments of Applied Mathematics and Polymer Engineering, The University of Akron, \\ Akron, $\mathrm{OH}, \mathrm{USA}$ \\ Email: leonov@uakron.edu
}

Received 4 April 2014; revised 11 May 2014; accepted 30 June 2014

Copyright ( 2014 by author and Scientific Research Publishing Inc.

This work is licensed under the Creative Commons Attribution International License (CC BY).

http://creativecommons.org/licenses/by/4.0/

(c) () Open Access

\begin{abstract}
A two-layer theoretical model of hurricanes traveling (quasi-) steadily over open seas has been developed. The use of coherency concept allowed avoiding the common turbulent approximations, except a thin sub-layer near the air/sea interface. The model analytically describes 3D distributions of dynamic and thermodynamic variables in hurricanes and analyzes processes of evaporation and condensation. Using this modeling, the following fundamental problems were naturally resolved-change in the cyclonic/anti-cyclonic directions of hurricane rotation and the directions of radial wind in lower and upper parts of hurricane; increase in wind angular momentum in hurricane boundary layer; dramatic effect of ocean spray and its radial distribution; and a high increase in temperature at the upper region of boundary layer. Additionally, integral balances allowed expressing the governing parameters of field variables via two external parameters, the sailing wind and temperature of a warm air band, in which direction the hurricane travels. A rude model for the hurricane genesis and maturing has also been developed.
\end{abstract}

\section{Keywords}

Hurricane, Aerodynamics, Adiabatic and Boundary Layers, Air-Sea Interaction

\section{Introduction}

The hurricanes (typhoons) have been extensively investigated during the last 60 years. Many of their features have been observed and experimentally studied using satellites, aircrafts, ships, and buoys. These observations created a detailed qualitative picture of hurricane structure, documented in several well-known texts by Dunn [1], Anthes [2], Hsu [3], Cotton \& Anthes [4], Ogawa [5], and Emanuel [6].

Some idealized models [7]-[12] of several problems in hurricanes have also been developed. Complicated role of mesovortices in the hurricane eye was experimentally modeled in laboratory and discussed [13]. Lighthill developed a thermodynamic theory of ocean spray [14], and its effect on the dynamics of near water air turbu- 
lence was revealed by Barenblatt et al. [15]. Detailed models of coupled interactions between the turbulent wind and oceanic waves near the air/sea interface have also been elaborated in text [16] (Ch. 3). Other conceptual ideas are mixed with numerical studies. Some works [17]-[20] modeled intriguing aspects of hurricane maturing. Many other papers developed turbulent baroclinic and barotropic numerical models (e.g. see paper [21] and references there). To forecast hurricane travel these models interact with the current synoptic and lower scale observations (see recent extensive reviews in Refs. [22] [23]).

Yet several fundamental problems in hurricane physics remain unresolved. These are the change in the directions of hurricane rotation and radial wind in lower and upper parts of hurricane, radial increase in wind angular momentum in hurricane boundary layer, dramatic effect of ocean spray and its radial distribution, and a high increase in temperature at the upper region of boundary layer. The problems of hurricane genesis and maturing are also currently vaguely addressed.

Thus the main objective of this paper is to resolve the above problems by developing and analyzing some quantitative models, based on the author's results [24]-[27] pre-published in Arxive. The models being rude enough still provide a consistent analytical description of the basic physical phenomena in hurricanes. The conceptual view of hurricanes as coherent structures, allows avoiding the common turbulent approximations except friction factors at the air/sea interface. The use in the model the aerodynamics of ideal gas requires implementing continuity for dynamic variables to avoid the Kelvin-Helmholtz (K-H) instability. Additionally, integral balance equations allow expressing all parameters in the distributions of field variables via only two external parameters - the sailing wind and temperature of warm air band the hurricane travels along.

The paper is organized as follows. The next Section briefly discusses the external forces causing horizontal travel of hurricanes, thermodynamics of air, dynamics of ideal liquids, and hurricane structure. Section 3 models the basic airflows in the upper layer of hurricane. Section 4 models the basic processes in the hurricane boundary layer. The last, Section 5 presents simple analytical models for hurricane genesis and maturing.

\section{Preliminaries}

\subsection{Horizontal Travel of Hurricanes}

Two factors affect the horizontal travel of hurricane: 1) stirring or "sailing" wind with velocity $\underline{w}_{s}$ and 2) "affinity” motion with velocity $\underline{w}_{a}$ because of hurricane’s tendency for accepting warmer air from environment. The value $w_{a}$ is unknown and should be found with solving problem. The additivity principle $\underline{w}=\underline{w}_{s}+\underline{w}_{a}$ holds for describing horizontal hurricane travel.

\subsection{Aerodynamic Equations for Air Flows}

We consider air motions in hurricane as axially symmetric flows of ideal compressible gas. The frame of reference used below is a cylindrical coordinate system with vertical $Z$-axis directed upward, slowly traveling in a horizontal direction. The axially symmetric motions of air relative to the local Earth rotation have very well known form (e.g. see Equations (1.7)-(1.10) in [24]). In the stationary cases, these equations yield two first integrals, which present the angular momentum $M$ and temperature $T$ as arbitrary functions of the stream function $\Psi$.

\subsection{Thermodynamics of Humid Air}

Far away from hurricane, the atmosphere is assumed to be horizontally homogeneous with vertically distributed ambient density $\rho_{a}$ pressure $p_{a}$ and temperature $T_{a}$. These distributions are described by the equilibrium equations within the thermodynamics of humid ideal gas [28].

Using adiabatic description of air,

$$
p_{a}=p_{a}^{0}\left(\rho_{a} / \rho_{a}^{0}\right)^{\gamma}
$$

and the static equation,

$$
\mathrm{d} p_{a} / \mathrm{d} z=-\rho_{a} g,
$$

the vertical distributions of thermodynamic parameters are presented as: 


$$
\rho_{a}(z) / \rho_{a}^{0}=\psi^{\frac{1}{\gamma-1}}, \quad p_{a}(z) / p_{a}^{0}=\psi^{\frac{\gamma}{\gamma-1}}, \quad T_{a}(z) / T_{a}^{0}=\psi, \quad \psi(z) \equiv 1-\frac{(\gamma-1) g z}{\gamma R T_{a}^{0}} .
$$

Here $p_{a}^{0}=\rho_{a}^{0} R T_{a}^{0}$ with $p_{a}^{0}, \rho_{a}^{0}$ and $T_{a}^{0}$ being ambient parameters at the surface, $\gamma=c_{p} / c_{\mathrm{v}} \approx 1.4$ is the adiabatic index, and $c_{p}$ and $c_{\mathrm{v}}$ are the heat capacities at constant pressure and constant volume, respectively.

\subsection{Hurricane Structure and Basic Processes}

A typical structure of a mature hurricane traveling (quasi-) steadily over the open sea is sketched in Figure 1. The hurricane is viewed as a solitary vertical air vortex rotating in the cyclonic direction near the bottom with additional radial and vertical air flows. It has a central "eye", a vertical column of radius $r_{i} \sim 20 \mathrm{~km}$, surrounded by the "eye wall" (EW) layer with external radius $r_{e}$ of $\sim 30-50 \mathrm{~km}$. Above the hurricane boundary layer (HBL) with vertical thickness $h_{b}$, the radius $r_{e}$ of external EW changes with height $z$. Along with a radial air flow, the air within the EW performs intense rotation, whose peak is achieved at $r_{e}$. An upward weak vertical speed component of airflow is mostly contained within the EW region. We call this rotating and ascending airflow as $E W$ jet. In the external region, outside the $\mathrm{EW}$, the relative rotation of hurricane decreases to zero at the external hurricane radius $r_{\text {ext }} \sim 400-600 \mathrm{~km}$. The radial airflow is inwards at the bottom and outwards at the top of hurricane. The entire vortex could be vertically layered into the bottom HBL, and upper "adiabatic" layer, with the total hurricane height up to 20 - $30 \mathrm{~km}$.

The vertical structure employed in the following models, includes the turbulent boundary sub-layer of thickness $h_{T} \sim 50-250 \mathrm{~m}$, HBL of height $h_{b} \approx(1.5-2) \mathrm{km}$, and very high "adiabatic" layer ascended up to troposphere. The affinity motion (if exists) is driven by environmental near-sea warm air band with the temperature $T_{+}$, which supplies a warmer air to the hurricane boundary layer. The geometry of the warm air band is simplistically viewed as a parallelepiped of height $h_{b}$ and width $H$. When $H \sim 2 r_{e}$, hurricane optimally adjusts the bottom EW size to the warm air band size.

In the HBL, the air-sea interaction directly affects the dynamics at the air/sea interface, generating oceanic waves which in turn interact with air flows in the outer part of HBL. There is also evaporation and the heat/mass exchange between the hurricane and environment. The moisture, sensible and latent heats are transported via HBL towards the EW jet. The height of HBL is limited by air moisture condensation, which causes the formation of spiral rain bands, layered clouds and rainfall from them. Dynamic effects of rainfall can seemingly be neglected, though the rainfall can balance the evaporation from the oceanic surface. This results in a constant salinity level in the oceanic boundary layer.

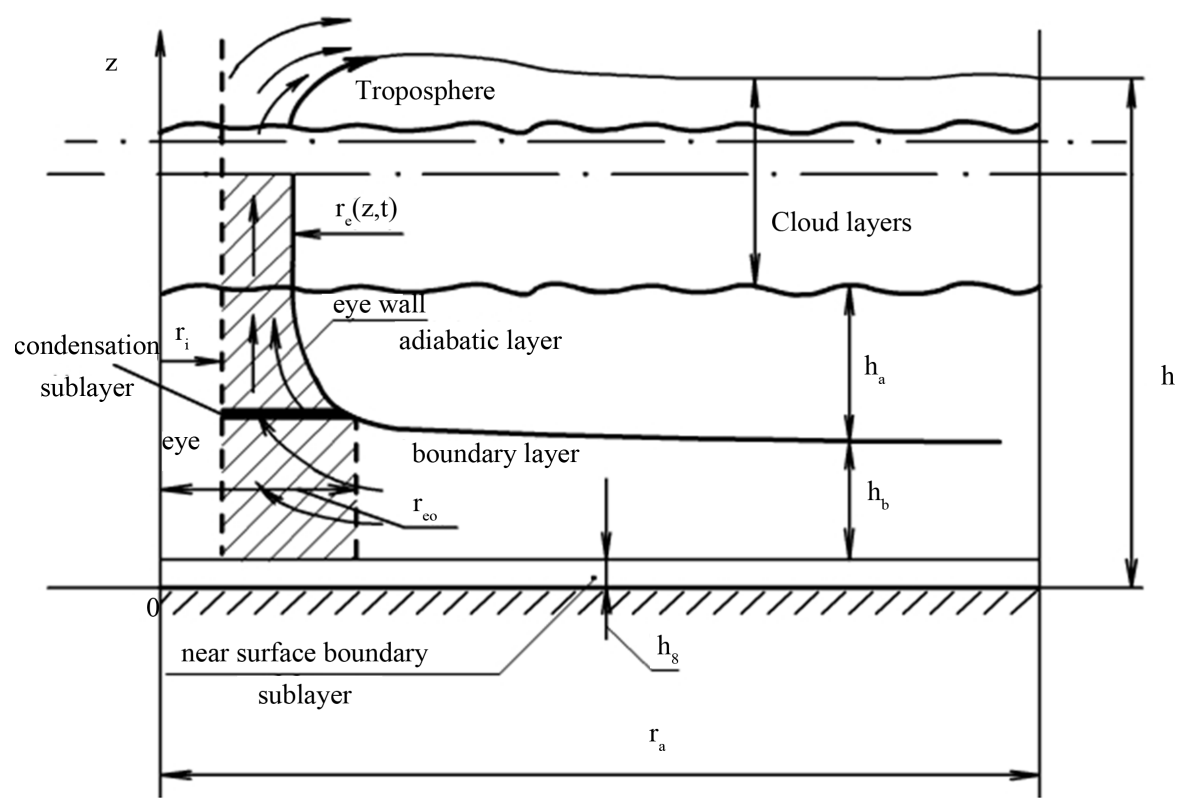

Figure 1. Schematic structure of a hurricane. 


\section{Model of Adiabatic Layer for Steady Hurricane [25]}

Neglecting the air band effects, air flows in upper layer of hurricanes can be modeled using the adiabatic approximation. The structure and basic flows in the hurricane adiabatic layer is sketched in Figure 2, and the airflows there are axi-symmetric. Here is a solid-like rotation of air in the eye region, and no vertical wind component exists in the outer region of hurricane. For convenience, we use in this Section the vertical axis $z$ shifted upward by the height $h_{b}$ of boundary layer.

The following modeling equations are used below [25]:

$$
\begin{gathered}
\partial_{r}\left(\rho r u_{r}\right)+\partial_{z}\left(\rho r u_{z}\right)=0, \quad \partial_{r}\left(\rho r u_{r} M\right)+\partial_{z}\left(\rho r u_{z} M\right)=0, \quad \chi=(\omega-\Omega)^{2} \\
\partial_{r}\left(\rho r u_{r}^{2}\right)+\partial_{r} p \approx \rho \chi r^{2}, \quad M=r\left(u_{\varphi}+\Omega r\right)=r^{2}(\omega+\Omega) \\
\frac{\mathrm{d}}{\mathrm{d} z} \int_{r_{i}}^{r_{e}} 2 r u_{z} \rho \mathrm{d} r=0, \quad 2 \frac{\mathrm{d}}{\mathrm{d} z} \int_{r_{i}}^{r_{e}} r u_{z}^{2} \rho \mathrm{d} r=\frac{\mathrm{d}}{\mathrm{d} z}\left(p_{a} \Delta s-2 \int_{r_{i}}^{r_{e}} r p \mathrm{~d} r\right)+g\left(\rho_{a} \Delta s-2 \int_{r_{i}}^{r_{e}} r \rho \mathrm{d} r\right) \\
\text { Here } r_{i}<r<r_{e}(z), \quad r_{e}^{\prime}(z)=\left(u_{r} / u_{z}\right)_{r=r_{e}(z)}, \quad \Delta s=r_{e}^{2}-r_{i}^{2}
\end{gathered}
$$

In aeromechanical Equations (4), $\omega$ is the angular air velocity relative to the angular velocity $\Omega$ of Earth rotation on $\beta$-plane. Equations (5) represent the "jet approach" [29] for vertical mass balance and momentum in the eye wall averaged over radius. Introducing the stream function by common relations, $\rho r u_{r}=-\partial_{z} \psi$, $\rho r u_{z}=\partial_{r} \psi$, yields the first integrals. Their linear forms $M=\alpha_{1} \psi+\alpha_{2}, T=\beta_{1} \psi+\beta_{2}$ with numerical coefficients $\alpha_{k}$ and $\beta_{k}$ allow an easy physical interpretation.

We now introduce two simplifying approximations:

$$
\text { (i) } u_{z} \approx u(z) \text {; (ii) } \rho \approx \rho_{a}(z)=\rho_{a}^{0}\left[\left\langle T_{a}(z)\right\rangle / T_{a}^{0}\right]^{2.5},\left\langle T_{a}(z)\right\rangle / T_{a}^{0}=1-\frac{g z}{3.5 R T_{a}^{0}}
$$

Here (6i) presents the "well mixing” assumption introduced by Deppermann [30], and independency from air rotation introduced in (6ii) has been justified in Ref. [25].

It is convenient to introduce the non-dimensional variables:

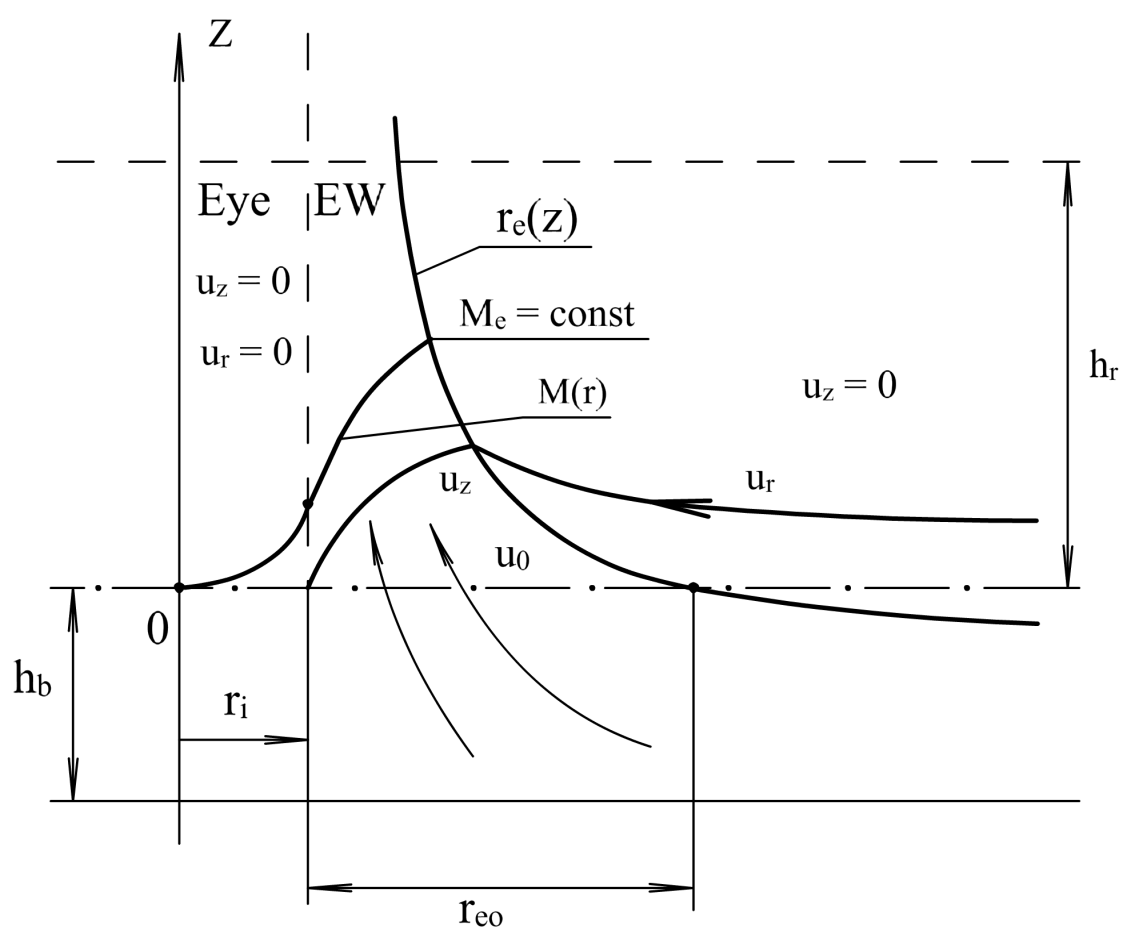

Figure 2. Sketch of adiabatic layer. 


$$
\begin{aligned}
& \hat{z}=\frac{g z}{3.5 R T_{a}^{0}}, \hat{r}=\frac{r}{r_{i}}, s=\hat{r}^{2}, \quad \hat{r}_{a}=\frac{r_{a}}{r_{i}}, \quad \hat{r}_{e}(z)=\sqrt{q(z)+1}, \hat{M}=\frac{M(r, z)}{M_{e}}, \hat{\omega}=\frac{\hat{M}}{\hat{r}^{2}}, \hat{\Omega}=\frac{r_{i}^{2} \Omega}{M_{e}} \\
& \hat{u}_{\phi}=\frac{u_{\varphi} r_{i}}{M_{e}}, \quad \hat{u}_{z}=\frac{u_{z}(z)}{\sqrt{R T_{a}^{0}}}, \quad \hat{u}_{r e}=\hat{u}_{z} r_{e}^{\prime}(z), \quad \hat{u}_{r}=\frac{u_{r}(r, z)}{\sqrt{R T_{a}^{0}}}, \hat{p}=\frac{p(r, z)}{p_{a}^{0}}, \hat{T_{a}}(\hat{z})=\frac{T_{a}(z)}{T_{a}^{0}}=1-\hat{z}
\end{aligned}
$$

Here $u_{r e}=u_{z} r_{e}^{\prime}(z)$ is the kinematic condition which relates the vertical and radial velocity at the outer jet radius. The common boundary conditions of continuity are employed for radial and rotational components of air field, with "frictional" kinks in distribution of angular momentum at the inner and outer walls of EW. Other natural conditions used in calculations are: $\rho \rightarrow \rho_{a}(z), p \rightarrow p_{a}(z)$ when $r \gg r_{e}(z)$.

Tedious calculations of set (4) with approximations (6) yield the explicit expressions for radial non-dimensional distributions of dynamic variables:

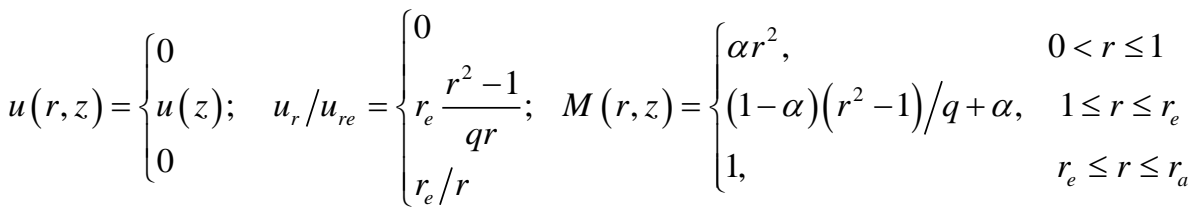

$$
\begin{aligned}
& u_{\varphi}(r, z)=M(r, z) / r-\Omega r \approx M(r, z) / r, \omega_{e}(z)=1 /(q+1)^{2}-\Omega
\end{aligned}
$$

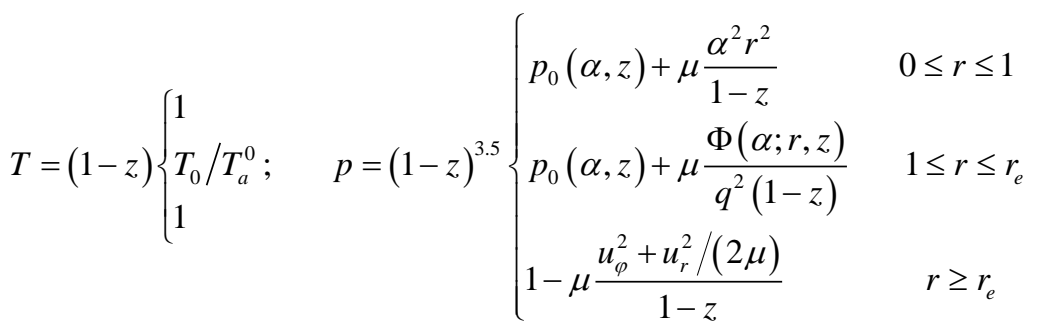

$$
\begin{aligned}
& q(\hat{z})=r_{e}^{2}(z) / r_{i}^{2}-1 ; \mu=M_{e}^{2} /\left(2 r_{i}^{2} R T_{0}\right) ; \quad \alpha=\left(r_{i} / r_{e 0}\right)^{2} / 2
\end{aligned}
$$

The approximation $\omega_{e} \gg \Omega$ or $r \ll r_{e x t}$ was used in formulas (8). Here $r_{e x t} \approx \sqrt{r_{e 0} u_{\varphi \max } / \Omega}$ is the external radius of hurricane. In (8), parameter $\mu$ characterizes the effect of rotation on the EW jet cross-section, and parameter $\alpha$ describes the frictional kink in the distribution of angular momentum at $r=r_{i}$ [25]. The structural functions $p_{0}(\alpha, z)$ and $\Phi(\alpha ; r, z)$ are given by (A1) in Appendix.

Formulas (8) show that streamlines in hurricane are the circles in eye and outside EW, and ascending spirals in EW with kinks at $r=r_{i}, \quad r=r_{e}(z)$. Equations for jet radius and vertical velocity profile are then found upon substituting (8) into (5). It yields the equations for mass conservation, and evolution of the jet profile. These equations are written in the non-dimensional form (7) as:

$$
q(z) u(z) \chi^{2.5}=q_{0} u_{0} \chi_{0}^{2.5} ; \frac{\mathrm{d} q}{\mathrm{~d} z}=\frac{q}{\chi} \cdot \frac{2.5 u_{0}^{2} q_{0}^{2} \chi_{0}^{5}+2.5 \mu q F_{\alpha}(q) \chi^{5}-\tau_{0} q^{2} \chi^{6}}{u_{0}^{2} q_{0}^{2} \chi_{0}^{5}+\mu q^{2} F_{\alpha}^{\prime}(q) \chi^{5}} ;\left.q\right|_{z=h}=q_{0}
$$

Here $\chi=1-z, \tau_{0}=3.5\left(T_{0} / T_{a}^{0}-1\right)$ is the buoyancy parameter, and $q(\hat{z})=r_{e}^{2}(z) / r_{i}^{2}-1$. The values $u_{0}, q_{0}$ and $\chi_{0}$ in (9) correspond to $z=h$, the structural function $F_{\alpha}(q)$ is given by (A2) in Appendix, and $F_{\alpha}^{\prime}(q)=\mathrm{d} F_{a} / \mathrm{d} q$.

There are simple asymptotic solutions of (9) in two limiting cases.

$$
\text { 1) } z \rightarrow h: q(z) \approx q_{0} \exp \left[-(z-h)\left(\tau_{0}-\tau_{1}\right) / \tau_{2}\right]\left(\tau_{1}, \tau_{2} \sim \mu, 0<\tau_{2} \ll \tau_{1}\right)
$$

Here $\tau_{k}$ are some algebraic functions of $q_{0}$ and $u_{0}$ presented via formula (A2) [25]. The only physically feasible case is $\tau_{0}>\tau_{1}$ where for stability, the heat supply from HBL to the hurricane jet should exceed the adiabatic cooling. Here the initial jet profile is convex down, with centripetal radial flow $u_{r}<0$.

$$
\text { 2) } z \rightarrow 1: \quad q=c_{1}[(1-h) /(1-z)]^{2.5 v} ; u_{m}=\rho u=c_{2} / q \quad\left(v=(1-h)^{5}\right)
$$


When $z \rightarrow 1$, the vertical component of air flows vanishes being converted to the radial one, with the jet radius approaching to infinity.

It was found that the numerical solution of steady problem (9) exists only for physically feasible case $\tau_{0}>\tau_{1}$. The following realistic parameters were accepted below in the demonstrative calculations: $r_{e 0}=40 \mathrm{~km}$, $r_{i}=20 \mathrm{~km}, \quad u_{\varphi e}^{0}=50 \mathrm{~m} / \mathrm{s}, \quad p_{a}^{0}=1000 \mathrm{mb} ; \Omega=5 \times 10^{-6} \mathrm{~s}^{-1}, \quad u_{0} \approx 0.0102$ or $0.00363(\sim 3$ or $1 \mathrm{~m} / \mathrm{s})$, $R \approx 2.9 \times 10^{2} \mathrm{~J} /(\mathrm{K} \cdot \mathrm{kg}), \quad T_{a}^{0} \approx 3 \times 10^{2} \mathrm{~K}, \quad \tau_{0}=0.07 \quad\left(T_{0}=33^{\circ} \mathrm{C}\right)$.

The values of calculated parameters are: $\alpha=1 / 8, h=0.0242, q_{0}=3, \quad \mu=0.0574, \tau_{1} \approx 0.0598$, $\tau_{2} \approx 6.30 \times 10^{-4}, r_{\text {ext }} \approx 632 \mathrm{~km}$, and $M_{e}=2 \times 10^{6} \mathrm{~m}^{2} / \mathrm{s}$.

Figures 3-9 illustrate the calculated radial distributions of basic variables, depending on altitude and initial value of vertical velocity $u_{0}$. Figure 3 demonstrates the characteristic non-monotonic behavior of outer EW jet boundary, Figure 4 the increase in vertical velocity. Figure 5 shows that the angular velocity in EW jet decreases with altitude, though the region when it is negative is not shown. Radial distributions of angular momentum $M$ and tangential velocity $u_{\varphi}$ for two altitudes, presented in Figure 6 and Figure 7, demonstrate their increase in the eye and EW regions with two characteristic kinks, a plateau for $M$ outside EW jet, and a sharp peak of $u_{\varphi}$ at the outer boundary of EW. Figure 8 show characteristic distributions of radial velocity. It is

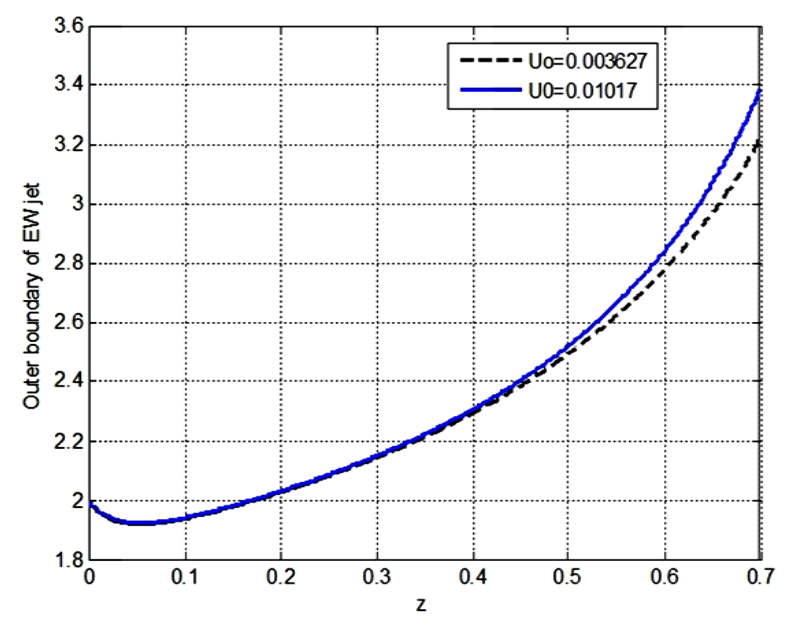

Figure 3. Non-dimensional altitude dependence of outer boundary of EW jet $r_{e}(z) ; u_{0}=0.01017$ (solid line) and 0.003627 (dashed line).

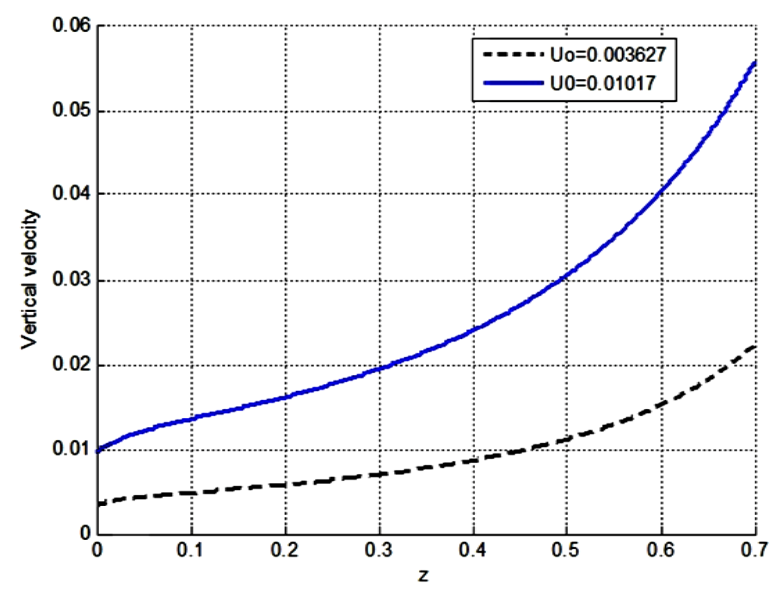

Figure 4. Non-dimensional altitude dependence of vertical velocity $u(z)$ in the EW jet. Parameters are the same as in Figure 3. 


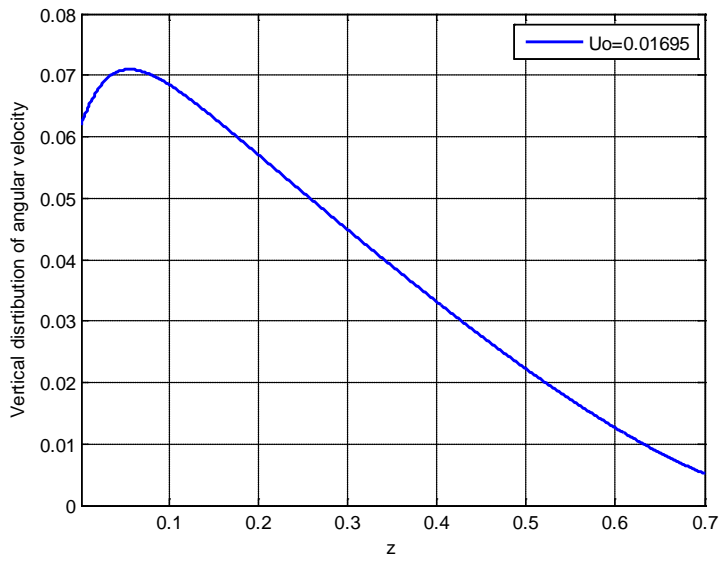

Figure 5. Vertical distribution of non-dimensional angular velocity $\omega_{e}(z)$ of EW jet.

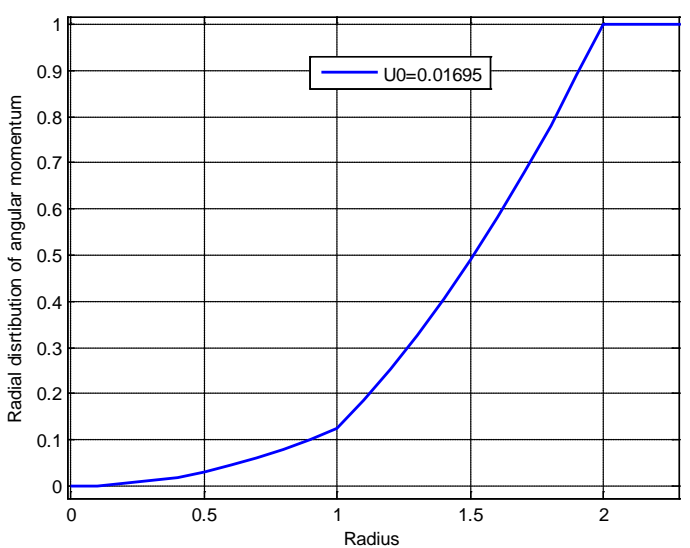

(a)

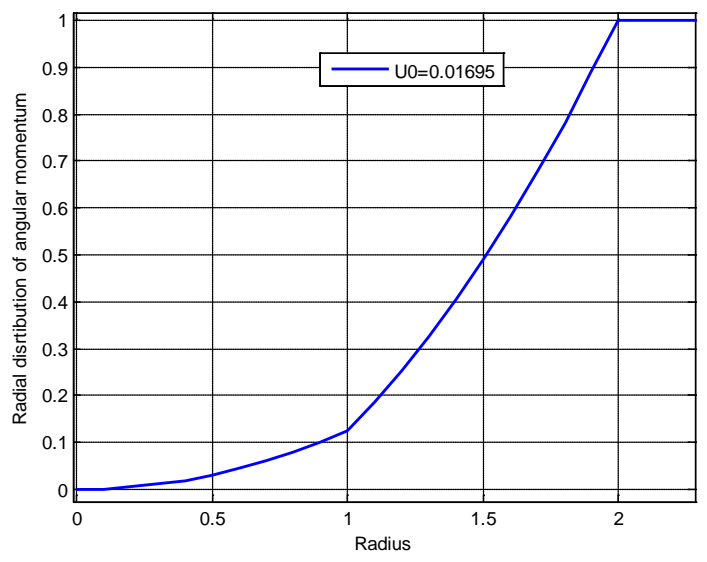

(b)

Figure 6. Non-dimensional radial distributions of angular momentum $M(r, z)$ at two non-dimensional altitudes: (a) $z=h$; (b) $z=0.3$.

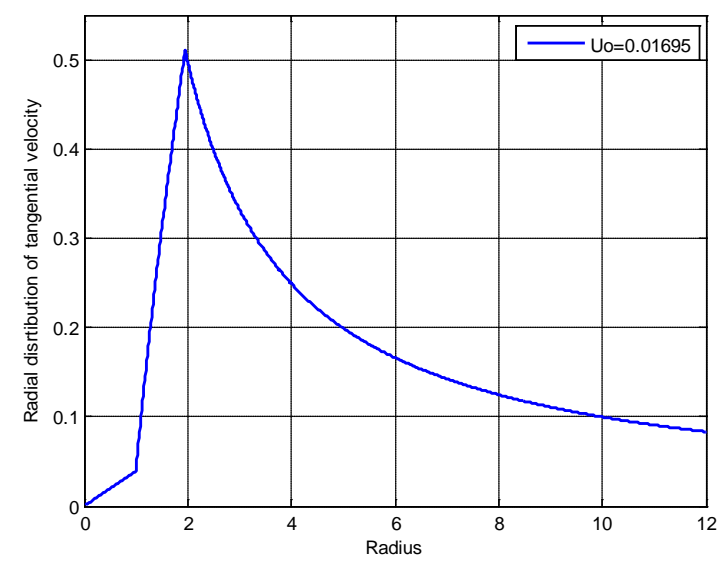

(a)

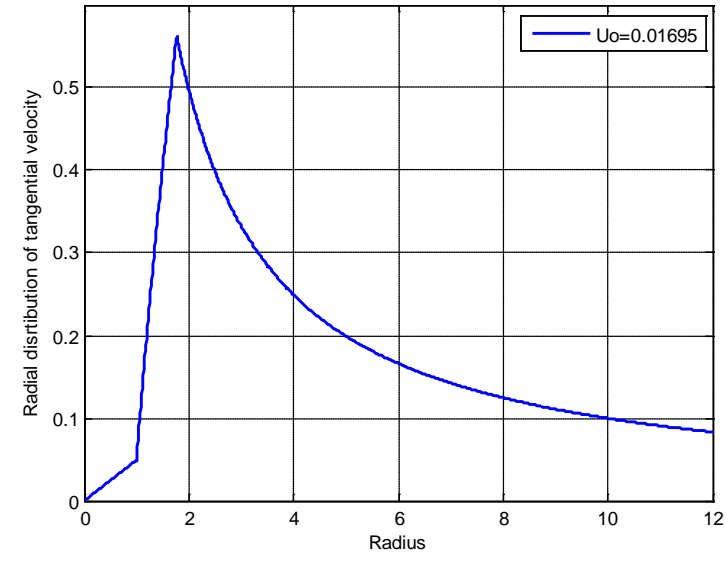

(b)

Figure 7. Non-dimensional radial distributions of tangential velocity $u_{\varphi}(r, z)$ at two non-dimensional altitudes: (a) $z=h$; (b) $z=0.3$. 
negative (centripetal) at lower and positive at higher altitudes, with absolute maximum at the outer boun dary of EW jet. Finally, Figure 9 show two similar radial distributions of pressure which display a characteristic "depression" area at the center of hurricane. These results support a well-documented characteristic structure of hurricane sketched in Figure 10. Additionally, the radial distributions of tangential velocity and pressure at the bottom of adiabatic layer were found in [25] in a good agreement with these obtained using semi-empirical modeling and observation data by Deppermann [30].

\section{Modeling the Boundary Layer in Steady Hurricane [26]}

The structure and basic interactions in hurricane boundary layer (HBL) are sketched in Figure 11. Here the HBL is horizontally separated in the same three regions as in previous Section: the eye, HBL EW, and outer HBL region, the latter having generally a curvilinear upper surface. There are two major vertical sub-sections in HBL: upper aerodynamic and lover turbulent ones. Additionally, there is a condensation sub-layer located at the top of HBL EW and assumed to be very thin $(\sim 100 \mathrm{~m})$. The height $h_{e}$ of HBL is restricted to the condensation level $h_{c}$ whose value is roughly evaluated using an empirical condition for the beginning condensation when the saturation point is achieved $h_{c} \approx 0.12\left(T_{-}-T_{D 0}\right)(\mathrm{km})$ [4]. Here $T_{-}-T_{D 0}$ is the dew point temperature

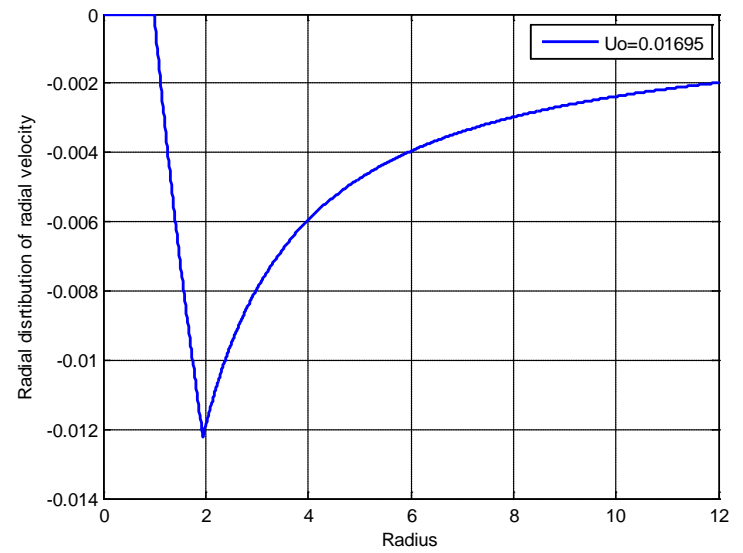

(a)

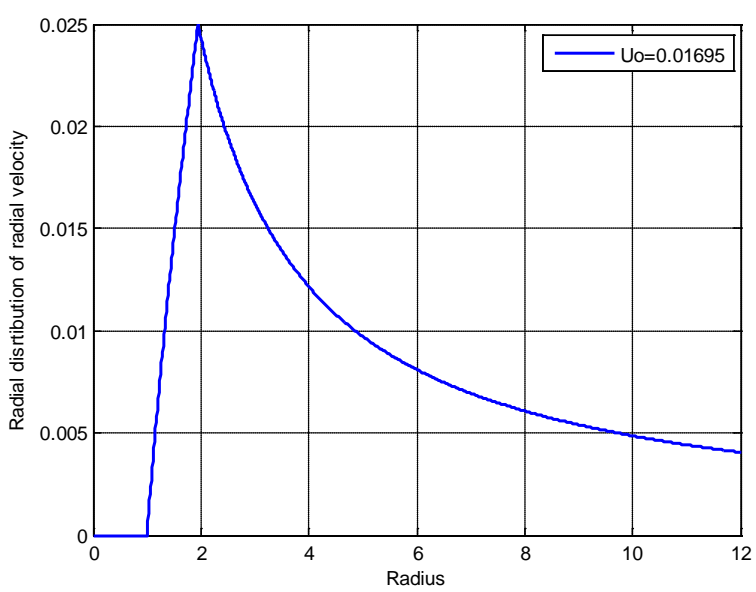

(b)

Figure 8. Non-dimensional radial distributions of radial velocity $u_{r}(r, z)$ at two non-dimensional altitudes: (a) $z=h$; (b) $z=0.3$.

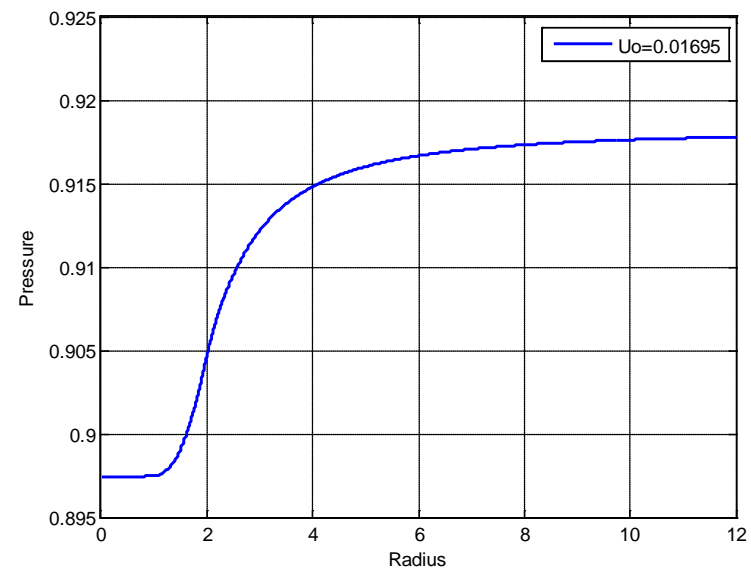

(a)

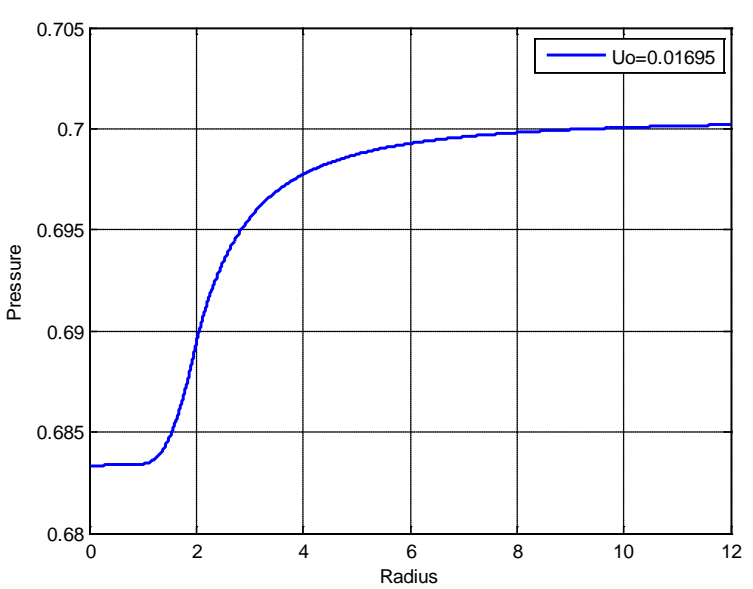

(b)

Figure 9. Non-dimensional radial distributions of pressure $p(r, z)$ at two non-dimensional altitudes: (a) $z=h$; (b) $z=0.3$. 


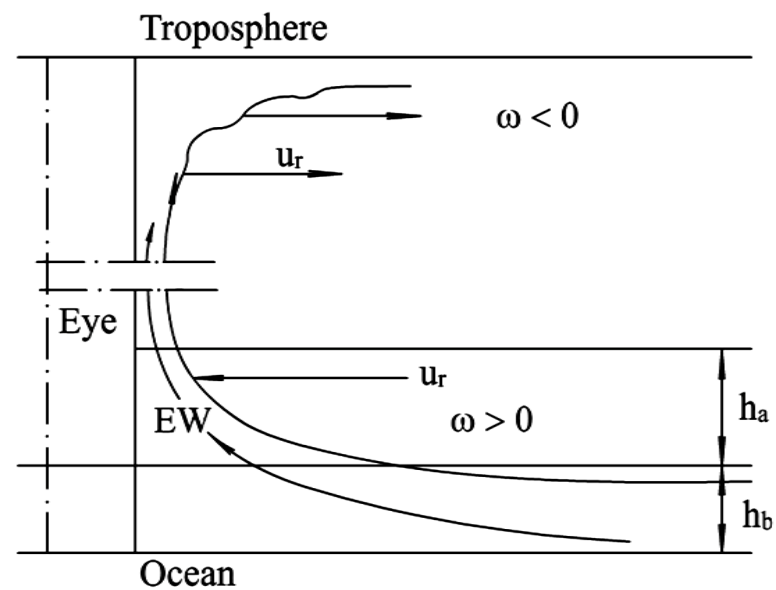

Figure 10. Sketch of the total vertical distribution of EW jet.

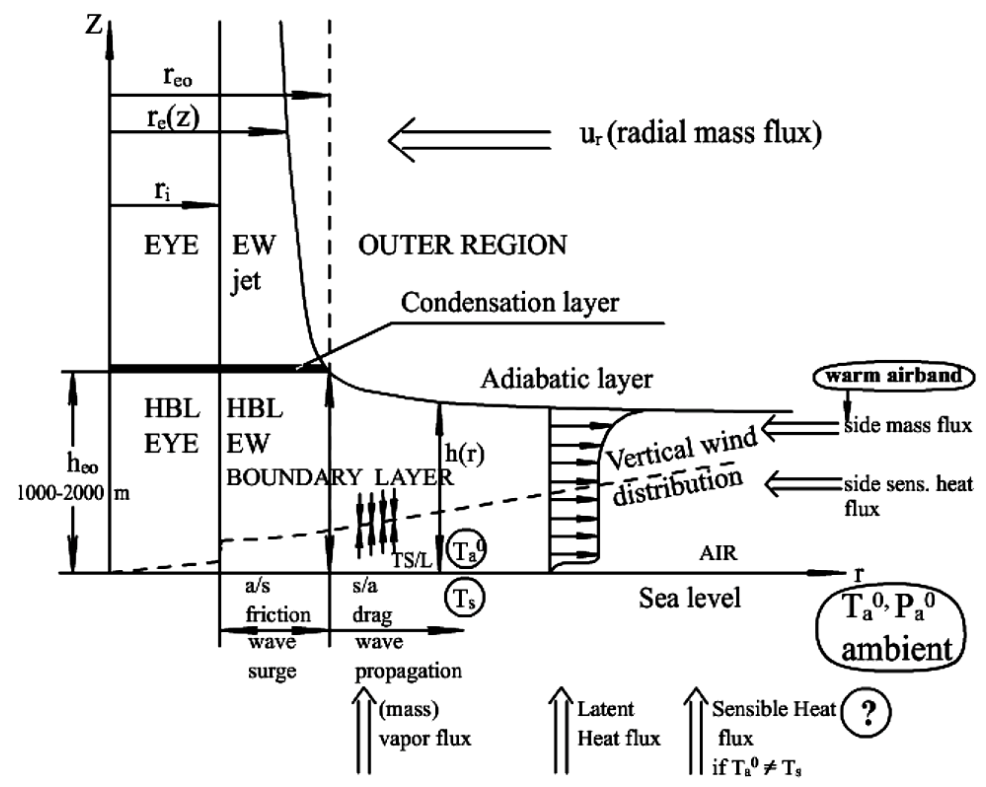

Figure 11. Cross-sectional sketch of HBL and diagram of air/sea interactions.

depression at the sea surface. The common evaluation $T_{-}-T_{D 0} \approx(10-15)^{\circ} \mathrm{C}$ yields $h_{c} \approx 1.2-1.8 \mathrm{~km}$.

\subsection{Fluid Mechanical Effects in HBL}

They include coherent aerodynamic airflows in upper part of HBL, turbulent airflows in lower part of HBL, and dynamic interaction of oceanic waves with HBL airflows.

\subsubsection{Aerodynamic Airflow}

Models employ simplified equations of aerodynamics of ideal gas similar to Equations (4) with $\rho \approx \rho_{a}^{0}$, but omitting the $\Omega$ effects:

$$
\begin{aligned}
& \partial_{r}\left(r u_{r}\right)+\partial_{z}\left(r u_{z}\right)=0, \quad \partial_{r}\left(r u_{r} M_{b}\right)+\partial_{z}\left(r u_{z} M_{b}\right)=0, \\
& u_{\varphi} \approx M_{b} / r ; \quad p(r, z) \approx \hat{p}(r, h)
\end{aligned}
$$

Omitting the $\Omega$ effects in (12) makes these equations inapplicable far away from the HBL EW. The last formula in (12) presents a typical boundary layer approximation. Here $\hat{p}\left(r, h_{b}\right)$ is the barometrically corrected 
radial pressure distribution at the bottom of adiabatic layer described at $z=h$ by Equation (8).

The same assumptions as in the previous Section are employed here: the rigid-like airflow in HBL eye, the radial independence of vertical wind in HBL EW, and the same boundary conditions at the inner HBL EW interface. It is also assumed that the outer upper boundary $h(r)$ of HBL is inpenetrable for the vertical wind component. Although far away from HBL EW the airflow is not axisymmetric, it is still modeled as pseudosymmetric one.

Introducing the stream function $\psi$ as $\rho r u_{r}=-\partial_{z} \psi$ and $\rho r u_{z}=\partial_{r} \psi$, yields two first integrals written in the linear form as: $M_{b}=\alpha_{1} \psi+\alpha_{2}, T=\beta_{1} \psi+\beta_{2}$. It is convenient to introduce here new non-dimensional coordinates, vertical $\eta=z / h_{e}$, and radial $\xi=r / r_{e}$ ones. Here $0 \leq \eta \leq 1$, and $\xi>\xi_{i}=r_{i} / r_{e}<1$. It is initially assumed that the angular momentum $M_{b} \sim \sqrt{\xi}$ at $\xi>1$, which will be justified later. Then the explicit solution [26] of set (12) is presented by for EW and outer region of HBL as:

$$
\begin{gathered}
\psi=U r_{e} h_{e} \frac{\xi^{2}-\xi_{i}^{2}}{1-\xi_{i}^{2}} f(\eta), \quad u_{r}=-\frac{\xi-\xi_{i}^{2} / \xi}{1-\xi_{i}^{2}} U f^{\prime}(\eta), \\
u_{z}=\frac{2 U h_{e} / r_{e}}{1-\xi_{i}^{2}} f(\eta), \quad M_{b}=\frac{\xi^{2}-\xi_{i}^{2}}{1-\xi_{i}^{2}}(1-\alpha) M f(\eta)+\alpha M, \\
u_{\varphi}=\frac{M}{r_{e}}\left[\frac{\xi-\xi_{i}^{2} / \xi}{1-\xi_{i}^{2}}(1-\alpha) f(\eta)+\frac{\alpha}{\xi}\right], \quad \xi_{i} \leq \xi \leq 1,0 \leq \eta \leq 1 \\
\psi(r, z)=U r_{e} h_{e} \sqrt{\xi} f(\eta), \quad u_{r}=-\frac{U}{\sqrt{\xi}} f^{\prime}(\eta), \\
u_{z}=\frac{h_{e}}{r_{e}} \frac{U}{2 \xi^{3 / 2}} f(\eta), \quad M_{b}=M(1-\alpha) \sqrt{\xi} f(\eta)+M \alpha, \\
u_{\varphi}=\frac{M(1-\alpha)}{r_{e} \sqrt{\xi}} f(\eta)+\frac{M \alpha}{r_{e} \xi}, \quad \xi>1,0<\eta<h(r) / h_{e}
\end{gathered}
$$

Here $\alpha=\left(r_{i} / r_{e 0}\right)^{2} / 2$ is the same frictional kink parameter and total radial air flow flux is:

$U=-\left(u_{r e}+w_{*}\right)>0$. Here $u_{r e}$ is the induced radial velocity at the lower boundary of adiabatic layer at $r=r_{e}$, and $w_{*}=w / \pi$ is a "pseudo-radial" contribution of hurricane travel speed $w$. The non-dimensional function $f(\eta)$ characterizing the vertical structure of velocity field cannot be determined using the ideal aerodynamics. It is assumed to be positive, slightly varied and monotonically increased $\left(f^{\prime}(\eta)>0\right)$. Formulas 13(a), 13(b) show that except vertical wind component $u_{z}$, two other wind components and angular momentum $M_{b}$ are continuous at the radial boundaries $\xi=\xi_{i}$ and $\xi=1$.

At the upper boundary HBL EW, $f(\eta)$ satisfies the natural boundary condition $f(1)=1$, which guaranties continuity for dynamic variables here. Also, since the upper boundary $z=h(r)$ of HBL is assumed to be impenetrable, the condition $\psi(r, h(r))=$ const defines a particular streamline separating the HBL from the adiabatic layer. The evident kinematical relation $u_{r} \mathrm{~d} h / \mathrm{d} r=u_{z}$ holds at $z=h(r)$. Since $u_{r}<0$ and $u_{z}>0$ in HBL the model predicts decreasing thickness of HBL towards periphery $h^{\prime}(r)<0$. Rewriting the boundary condition at $z=h(r)$ in the form $f(\hat{h}(\xi))=\xi^{-1 / 2}$, shows that the sharper increase in $f(\eta)$ the slower is $\hat{h}(\xi)$ decrease. The above results demonstrate that the streamlines in the HBL look like ascending spirals with ultimate streamline being the upper boundary $h(r)$ of HBL. Since at $z=h(r)$ both $M_{b}$ and $\Psi$ are continuous, the radial velocity component is also continuous at this boundary, although the vertical velocity component has a small jump there, similar to that found in the previous Section. It is also proven that the external boundary of EW jet smoothly continues downward, to the HBL upper boundary below the level $z=h_{e}$. Finally, at $Z=0$, we impose a rude condition $f(0)=0$, although at the lower level of HBL the aerodynamic model is invalid.

\subsubsection{Airflow in Turbulent Sub-Layer}

A huge air wind near the radius $r=r_{e}$ maintains a surge of broken oceanic waves under EW bottom with 
waves propagating outside this region. The radial wind contribution can be neglected in this sub-layer because of very low variation assumed for $f(\eta)$. Since no model currently exists for describing the interaction of airflow with broken oceanic waves, a semi-empirical approach is used below. It is based on the fact that at the anemometer height $z_{a}(\approx 10 \mathrm{~m})$ the horizontal wind speed is equal almost $75 \%$ of the air speed at the level of aircraft observation (see e.g. paper [31] and references there). This fact may happen because of direct dynamic effect of ocean spray [14] [15].

We use for friction factor $\tau_{e}$ the common bulk relation $\tau_{e}=\rho C_{D} u_{T}^{2}$ where $u_{T}$ is the mean velocity at the height $h_{T}$, yet to be established, and $C_{D} \sim 10^{-3}$ is the friction (drag) coefficient. The standard logarithmic profile is used for describing the mean velocity. It parameterized with roughness factor $z_{0}$ and reciprocal Karman constant $A \approx 2.5$. Matching the logarithmic profile with the aerodynamic profile (13) and using flatness of $f(\eta)$ yields [26]:

$$
u_{\varphi}(z, r) \approx A u_{\varphi}\left(h_{T}, \xi\right) \sqrt{C_{D}} \ln \left(z / z_{0}\right) \quad\left(z \geq z_{0}\right)
$$

Evaluation of the roughness factor $z_{0}$ and the height of turbulent sub-layer $h_{e T}$ at $\xi=1$ (or $r=r_{e}$ ) are presented in Table 1. Finally, extending the observation in paper [31] to the entire turbulent sub-layer of HBL and using (14), yields the distribution of tangential velocity at the anemometric height $z_{a}$ :

$$
u_{\varphi}\left(z_{a}, \xi\right) \approx 0.75\left(M / r_{e}\right)\left\{\begin{array}{lc}
{\left[(1-\alpha)\left(1-\xi_{i}^{2} / \xi\right) /\left(1-\xi_{i}^{2}\right)+\alpha / \xi\right],} & \xi_{i} \leq \xi<1 \\
{[(1-\alpha) / \sqrt{\xi}+\alpha / \xi],} & \xi>1
\end{array}\right.
$$

\subsubsection{Interaction of Air Wind and Oceanic Waves}

The radial increase in angular momentum $M_{b}$ as $\sim \sqrt{r}$ observed in the region $r>r_{e}$, was explained by Emanuel [7] who used some thermodynamic arguments, assuming that the height of HBL is constant. This assumption necessitates a vertical airflow through the upper HBL boundary. Another idea proposed by Dr. A. Benilov and elaborated by the author is presented below.

Consider the oceanic waves initiated in the vicinity $r=r_{e}$. They propagate into the outer area $r>r_{e}$ along the straight lines tangential to the circle $r=r_{e}$ with the constant phase speed $c_{e}$ (Figure 12). Therefore there is a skew interaction of the oceanic waves with air, resulted in dominant tangential airflow in the turbulent layer. Then the tangential shear stress $\tau$ along the wave path changes from the initial value $\tau_{e}=\tau_{r \varphi}\left(r_{e}\right)$ to the value $\tau_{r}=\tau_{r \varphi}(r)$ at the current radius $r$, as $\tau_{r} \approx \tau_{e} \sin \theta(r), \sin \theta(r)=r_{e} / r \quad\left(r \geq r_{e}\right)$. Using these formulas yields:

$$
\tau_{r}=\rho_{a}^{0} u_{*_{r}}^{2} ; u_{*_{r}} \sim c_{r}, \quad u_{*_{r}} \approx u_{*_{e}} \sqrt{r_{e} / r}, \quad c_{r} \approx c_{e} \sqrt{r_{e} / r}, \quad u_{\varphi}^{T} \sim u_{\varphi} \sim \sqrt{r_{e} / r} .
$$

Here the low indexes "e" and " $r$ " denote the values of variables at the radii $r_{e}$ and $r$, respectively, and $c_{r}$ is the local phase velocity of wave. Due to (16), $c_{r}$ is directed at the circle of radius $r$ under angle $\hat{\theta}(r)$ defined as: $\sin \hat{\theta}(r)=\sqrt{\sin \theta(r)}$. Hereafter the upper index " $T$ " denotes the values of variables in the turbulent sub-layer of HBL region 2. Formulas (16) explain the observed behavior of the tangential wind, and slightly differ from the second expression in (15). The result shows that in the turbulent sub-layer of outer HBL region, oceanic waves rather generate air wind than dissipate it. Since the ratio of air to water density is $\sim 10^{-3}$ the energy loss in wave to air transfer is negligible. Using then the wave energy conservation, results in the wave energy decay as $\sim 1 / r$.

\begin{tabular}{cccc|} 
Table 1. Values of roughness parameter & $z_{0}$ and height of turbulent boundary layer $h_{e T}$. \\
\hline$Z_{a}(\mathrm{~m})$ & $C_{D} \times 10^{3}$ & $Z_{0}(\mathrm{~m})$ & $h_{e T}(\mathrm{~m})$ \\
\hline 10 & 1 & 0.000511 & 270 \\
10 & 2 & 0.00923 & 103 \\
10 & 3 & 0.0333 & 70.0 \\
10 & 4 & 0.0714 & 51.9 \\
\hline
\end{tabular}




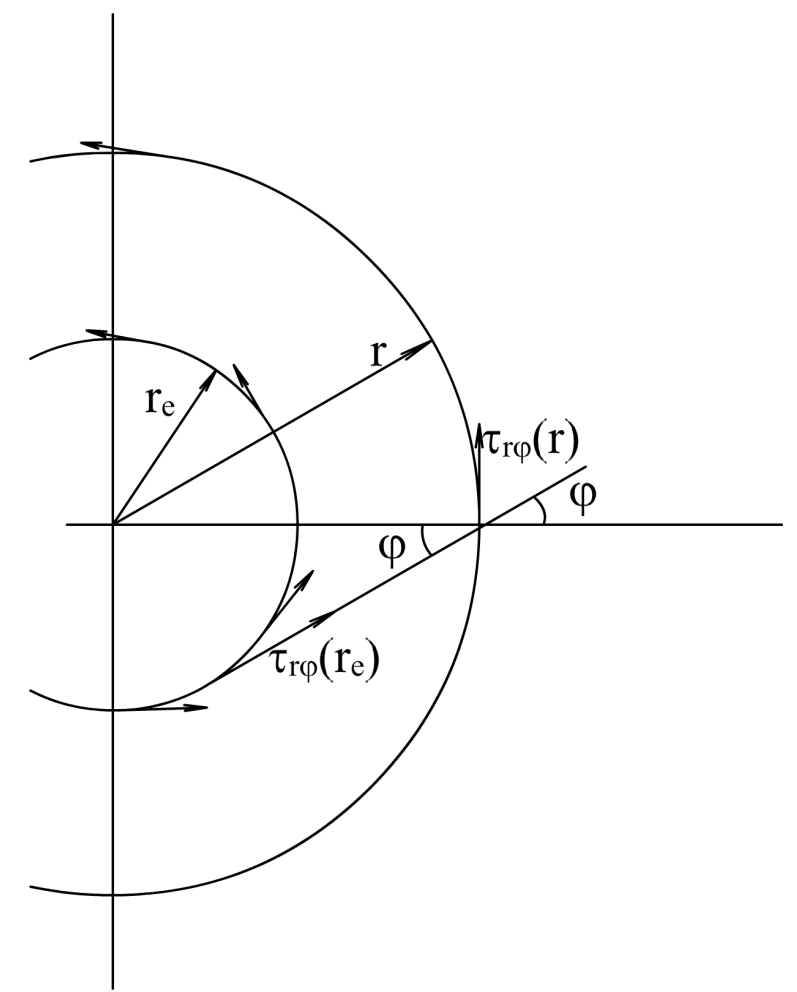

Figure 12. A sketch of skew interactions of oceanic waves with turbulent air in HBL.

\subsection{Physical Effects in HBL}

Evaporation from the oceanic surface and latent heat. Over calm oceanic water, the vertical air flux (per unit mass of vapor) caused by moisture evaporation can be approximated as $u_{\mathrm{v}}(r)=C_{E}\left|\underline{V}_{a}\right|$ [2]. Here $\underline{V}_{a}$ is the wind speed at the anemometer level $z_{a}(\sim 10 \mathrm{~m})$, and the exchange coefficient $C_{E} \sim 10^{-6}$. The ocean spray ejected over the oceanic water by the wave whitecaps, can increase the value of $u_{\mathrm{v}}(r)$ at least by an order of magnitude at the hurricane EW [14]. The studies [32] [33] found that whitecap concentration is $\sim\left|\underline{V}_{a}\right|^{m}$, where $m \approx 3-3.5$. We adopt here $m \approx 3$ as found in recent satellite observations. These results are incorporated in the model, under assumption that transfer coefficient $C_{E}$ is radial dependent decaying from its maximum value $C_{E m}$ at $r=r_{e}$ as a cube of relative velocity:

$$
C_{E}(\xi)=C_{E m}\left[u_{\varphi}\left(z_{a}, \xi\right) / u_{\varphi}\left(z_{a}, 1\right)\right]^{3} \text {, and } u_{\mathrm{v}}(r) \approx C_{E}(\xi) u_{\varphi}\left(z_{a}, \xi\right)
$$

Here $u_{\varphi}\left(z_{a}, \xi\right)$ is the near water wind speed given by (15), and for developed hurricanes $C_{E m}=C_{E}(1)$ is the maximum value of $C_{E} \sim 10^{-5}$ at $\xi=1$.

Consider an example. Using (15) with $\alpha=1 / 8$ and $r_{e}=40 \mathrm{~km}$, we find from (17) that at $\xi^{*}=5.25$ $\left(r^{*}=210 \mathrm{~km}\right)$ the value $C_{E}\left(\xi^{*}\right)$ is $\sim 15$ times lower than $C_{E}(1)$. It is in the range $r_{e x} \approx 150-350 \mathrm{~km}$ reported in Anthes book [2]. If the value of maximal tangential wind is $u_{\varphi m}\left(r_{e}\right)=50 \mathrm{~m} / \mathrm{s}$, Formula (17) shows that $u_{\varphi}\left(z_{a}, \xi^{*}\right) \approx 14.4 \mathrm{~m} / \mathrm{s}$, i.e. the process of effective evaporation continues in the entire hurricane area $\xi_{i}<\xi<\xi_{\text {ext }}$.

Using (15) and (17), the total mass flux of evaporation $Q_{\mathrm{v}}$ and the latent heat $Q_{L}$ are calculated as:

$$
Q_{\mathrm{v}} \approx 1.5 \pi \rho_{\mathrm{v}} u_{\varphi m} r_{e}^{2} C_{E m} \int_{\xi_{i}}^{\xi_{\text {ext }}} \hat{u}_{\varphi}^{4}\left(z_{a}, \xi\right) \xi \mathrm{d} \xi ; Q_{L}=Q_{\mathrm{v}} \Delta l \quad(l=L m) .
$$

Here $\rho_{\mathrm{v}}$ is the vapor density, $u_{\varphi m}=M / r_{e}$ is the maximal tangential wind speed, and $\hat{u}_{\varphi}\left(z_{a}, \xi\right)=r_{e} u_{\varphi}\left(z_{a}, \xi\right) /(0.75 M)$ is the non-dimensional value of the wind speed shown in (15). Also, in (18) 
$L$ is the specific latent heat of vaporization, $m$ is humidity, and $\Delta l$ is the value of latent heat excess over the environmental air at the sea surface.

Transfer of sensible heat is considered in the steady model under condition of complete sea/air temperature balance $T_{\mathrm{s}}=T_{a}^{0}$. Therefore direct heat exchange between ocean and HBL does not exist. It is in accord with the Chapter 3.9 of text [6] and in contradicts the assumption of the models [6] [23]. A small dissipative heat negligibly increases $T_{s}$ [26]. It means that in steady hurricanes the sensible heat could be transferred only from the horizontal warm air band.

Condensation is assumed to happen in a relatively thin vertical layer whose height is less than hundred meters, where the over-saturated vapor comes into the upper layer of HBL. Neglecting the thickness of the layer, it is considered as a weak condensation jump, which is described by basic equations including the conservation of vertical fluxes of mass, momentum and energy [34]. For a weak jump these equations are reduced to the continuity of mass flux, pressure and enthalpy on the jump interface, averaged over EW radius:

$$
\rho_{-} u_{-}=\rho_{0} u_{0}, \quad p_{-}=p_{0}, \quad E_{-}=c_{p} T_{-}+\langle m\rangle L=c_{p} T_{0}=E_{0}
$$

Since the differences between velocities, pressures and densities over the jump are negligible, $u_{0} \approx u_{-}$, $p_{0} \approx p_{-}, \rho_{-} \approx \rho_{0}$. Those simplifications are the same as in slow combustion theory [34].

\subsection{Integral Balances in the HBL Eyewall}

The average temperature $T_{0}$ and vertical velocity $u_{0}$ at the bottom of adiabatic layer are still unknown. Also generally unknown are the velocity $w$ of the horizontal motion of hurricane and the value of angular momentum $M$ in adiabatic layer. Using integral balances and given the geometrical structure of hurricane, these unknown parameters are expressed below through the known external parameters of hurricane, the sailing wind velocity $w_{s}$ and known temperature $T_{+}$of warm air band. This also allows disregarding the vertical structure of dynamic variables in HBL, described by the function $f(\eta)$.

In the following we use the plane Cartesian axes $x, y$; the axis $x$ coinciding with the axis of the warm air band. Then the parallel $(\|)$ and normal $(\perp)$ projections of the sailing and affinity components of horizontal wind are:

$$
w_{\|}=w_{s \|}-w_{a}, w_{\perp}=w_{s \perp} .
$$

\subsubsection{Mass Balance of Dry Air in HBL}

Two fluxes of the "dry" air masses come from HBL to the EW jet via the bottom of adiabatic layer: 1) the flux from the radial airflow into the HBL and 2) fresh air coming because of horizontal travel of hurricane. Neglecting density variations, the balance is:

$$
\pi\left(r_{e}^{2}-r_{i}^{2}\right) u_{0} \approx 2 \pi r_{e} h_{e}\left|u_{r e}\left(r_{e}\right)\right|+H h_{e}|w|,|w|\left|=\sqrt{\left(w_{s \|}+w_{a}\right)^{2}+w_{s \perp}^{2}}\right|
$$

Here $H \approx 2 r_{e}$ is the width of warm air band, $u_{r e}=u_{0} r_{r e}^{\prime}=-u_{0} k\left(\tau_{0}-\tau_{1}\right) / \tau_{2}$, where $\tau_{1}, \tau_{2} \sim u_{\varphi m}^{2}$ (see Equation(10) and references there). The left-hand side of (21) describes the air flux leaving the HBL; the first term in the right-hand side the rate of mass supplied by induced radial flow at the bottom of adiabatic layer, and the second is caused by horizontal hurricane motion.

\subsubsection{Balance of the Sensible Heat in HBL Reads}

$$
\pi\left(r_{e}^{2}-r_{i}^{2}\right) u_{0} \tau_{-} \approx H h_{e} w_{a} \cdot \tau_{+} \quad\left[\tau_{-}=3.5\left(T_{-} / T_{a}^{0}-1\right), \tau_{+}=3.5\left(T_{+} / T_{a}^{0}-1\right)\right]
$$

In (22) the differences between heat capacities are neglected. The left-hand side of (22) describes the heat entering the hurricane condensation layer with unknown temperature $T_{-}$, and the right-hand side the air heat supplied by the warm air band.

\subsubsection{Oceanic Vapor Mass Balance Is}

$$
Q_{\mathrm{v}}=\pi \rho_{\mathrm{v}}\left(r_{e}^{2}-r_{i}^{2}\right) u_{0}\langle m\rangle .
$$


Here $Q_{\mathrm{v}}$ is the mass flux of oceanic vapor presented by (18), and the right-hand side is over-saturated vapor flux into adiabatic layer from the condensation surface at $z=h_{c}$.

Balance of the latent heat is presented by second formula in (18).

Assuming that the oceanic vapor is completely condensed in the condensation layer, the last formula in (19) along with (23) yields two useful chain equalities:

$$
\langle m\rangle=\Upsilon C_{E}(1) u_{\varphi m} / u_{0}=c_{p}\left(T_{0}-T_{-}\right) / L_{\mathrm{v}} ; \Upsilon \approx \frac{1.5}{1-\xi_{i}^{2}} \int_{\xi_{i}}^{\xi_{\text {ext }}} \hat{u}_{\varphi}^{4}\left(z_{a}, \xi\right) \xi \mathrm{d} \xi .
$$

The values of $\Upsilon$, depending on parameters $\xi_{i}$ and $\xi_{\text {ext }}$, easily found numerically. E.g. $\Upsilon=4.214$ when $\xi_{i}=0.5$ and $\xi_{\text {ext }}=10$.

Entropy balance, detailed in [26], starts with the well-known equation:

$$
\partial_{r}\left(r u_{r} \Delta S\right)+\partial_{z}\left(r u_{z} \Delta S\right)=C_{D} u_{\varphi}^{3} / T_{a}^{0}
$$

Here entropy $\Delta S \approx c_{p} \tau / 3.5+L_{\mathrm{v}} m / T_{a}^{0}+R\left(p_{a}^{0}-p\right) / p_{a}^{0}$ is normalized on ambient conditions and the dissipation is localized at the sea-air interface in HBL EW. Integrating the above equation over the volume of HBL, except a thin bottom layer of EW of thickness $z_{a}$, and noticed that the latent and sensible heat had been balanced, the $\Delta S$ balance is reduced to the integral pressure balance. Tedious calculations yielded:

$$
u_{0}=\lambda u_{\varphi m} \quad\left(\lambda=\lambda_{0} C_{D}\right)
$$

Here numerical parameter $\lambda_{0}$ depends only on value of $\xi_{i}$, e.g. $\lambda_{0}=11.7$ for $\xi_{i}=0.5$.

Affinity velocity of hurricane travel was determined in [26], assuming the stream from warm air band is effectively mixed by dominant tangential air wind,

$$
w_{a}=v u_{\varphi m} \tau_{+}, \quad(v=2 \pi / 3.5 \approx 1.8)
$$

Thus, all the unknown parameters, $u_{0}, u_{\varphi m}, T_{-}$, and $T_{0}$ can be effectively found from the above equations with given values $w_{s}, T_{+}$, hurricane geometry, and parameters $C_{E}$ and $C_{D}$. Although the following calculations are explained in details in [26], these explanations are also briefly shown below because of some arithmetic mistakes and misspellings in the above report and new account of evaporation in this paper.

Recall that the non-dimensional temperatures $\tau_{+}, \tau_{-}$and $\tau_{0}$ are defined as:

$$
\tau_{+}=3.5\left(T_{+}-T_{a}^{0}\right) / T_{a}^{0}, \tau_{-}=3.5\left(T_{-}-T_{a}^{0}\right) / T_{a}^{0}, \tau_{0}=3.5\left(T_{0}-T_{a}^{0}\right) / T_{a}^{0} .
$$

It is convenient to introduce non-dimensional wind components, scaled with the adiabatic speed $\sqrt{R T_{a}^{0}}$ as $\left\{u_{0}, u_{\varphi m}, \underline{w}_{s}, w_{a}\right\}=\left\{u, \mathrm{v}, \underline{\hat{w}_{s}}, \hat{w}_{a}\right\} \sqrt{R T_{a}^{0}}$. Then (24) takes the form:

$$
\tau_{0}=\tau_{-}+(u / \mathrm{v}) \mathrm{X}, \quad\left(\mathrm{X}=3.5 \mathrm{r} C_{E} L /\left(c_{p} T_{a}^{0}\right)\right) .
$$

The above relations yield the five equations for parameters $\tau_{-}, \tau_{0}, u, \mathrm{v}$ and $\hat{w}_{a}$ :

$$
\begin{aligned}
& \lambda \mathrm{v}\left[1-\left(\gamma_{1} \tau_{-}+\gamma_{2} / \lambda\right) / \mathrm{v}^{2}+\gamma_{3}\right]=\varepsilon|\hat{w}| / \pi, \quad u=\lambda \mathrm{v} \\
& \tau_{-}=v \tau_{+}^{2}, \quad \tau_{0}=v \tau_{+}^{2}+\mathrm{X} / \lambda, \quad \hat{w}_{a}=v \mathrm{v} \tau_{+}
\end{aligned}
$$

Here $|\hat{w}|=\sqrt{\left(\hat{w}_{s \|}+\hat{w}_{a}\right)^{2}+\hat{w}_{s \perp}^{2}}, \quad q_{0}=\left(r_{e} / r_{i}\right)^{2}-1$, the functions $\varphi_{1}\left(q_{0}\right), \quad \varphi_{2}\left(q_{0}\right)$ are tabulated in report [25], and non-dimensional constants $\gamma_{n}, k, \varepsilon$, and $v$ are presented as:

$$
\begin{aligned}
& \gamma_{1}=\frac{2 k \varepsilon}{\left(1+q_{0}^{-1}\right) \varphi_{2}\left(q_{0}\right)}, \quad \gamma_{2}=\gamma_{1} \mathrm{X}, \quad \gamma_{3}=\frac{2.5 k \varepsilon \varphi_{1}\left(q_{0}\right)}{\varphi_{2}\left(q_{0}\right)}, \\
& \varepsilon=\frac{2 h_{e} / r_{e}}{1-\xi_{i}^{2}}, \quad k=\frac{g r_{i}^{2}}{7 R T_{a}^{0} r_{e}}, \quad v=\frac{v \varepsilon}{\lambda \pi}
\end{aligned}
$$

Substituting $|\hat{w}|$ in the first equation in (28) yields an awkward algebraic relation between tangential wind 
speed, and given values of horizontal temperature and sailing wind component. For illustrating purpose, only two limiting cases of this equation are considered below.

1) External sensible heat supply is negligible- $\tau_{+}=0$. The hurricane is only driven by sailing wind $\underline{w}_{s}$. Then $\tau_{-}=0, w_{a}=0,|\hat{w}|=\hat{w}_{s}$, and the dimensional solution is:

$$
\begin{aligned}
& u_{\varphi m}=\frac{\varepsilon w_{s}+\sqrt{\left(\varepsilon w_{s}\right)^{2}+4 R T_{a}^{0} \pi^{2} \lambda \gamma_{2}\left(1+\gamma_{3}\right)}}{2 \pi \lambda\left(1+\gamma_{3}\right)} ; \\
& u_{0}=\lambda u_{\varphi m} ;\left|u_{r e}\right|=\frac{u_{0}}{\varepsilon}-\frac{w_{s}}{\pi} ; \tau_{0}=X / \lambda
\end{aligned}
$$

One can see that due to (30) $u_{\varphi m}$ and $u_{0}$ are monotonically increasing functions of $w_{s}$, whereas $\left|u_{r e}\right|$ might decrease with $w_{s}$ growing.

2) Sailing wind is negligible $-w_{s}=0$. In this case the hurricane moves with affinity speed $w_{a}$, and the solution, presented in dimensional form is:

$$
u_{\varphi m}=\sqrt{R T_{a}^{0} \frac{\gamma_{1} v \tau_{+}^{2}+\gamma_{2} / \lambda}{1+\gamma_{3}-v \tau_{+}}} ; \quad u_{0}=\lambda u_{\varphi m} ; \quad w_{a}=v u_{\varphi m} \tau_{+} ; \quad\left|u_{r e}\right|=u_{0} \frac{1-v \tau_{+}}{\varepsilon} ; \quad \tau_{0}=v \tau_{+}^{2}+\frac{X}{\lambda}
$$

Formulas (31) show that $u_{\varphi m}, u_{0}, w_{a}$, and $\tau_{0}$ increase with $\tau_{+}$growing, while $\left|u_{r e}\right|$ might depend on $\tau_{+}$non-monotonously.

In the limit cases, $w_{s}=0$ in (30) and $\tau_{+} \rightarrow 0 \quad\left(w_{a}=0\right)$ in (31) the solution is:

$$
u_{\varphi m}^{0}=\left.u_{\varphi m}\right|_{w_{s}=0}=\sqrt{\frac{R T_{a}^{0} \gamma_{2}}{\lambda\left(1+\gamma_{3}\right)}} ; \quad u_{0}=\lambda u_{\varphi m}^{0} ; \quad\left|u_{r e}\right|=\frac{u_{0}}{\varepsilon} ; \quad \tau_{0}=\frac{X}{\lambda} .
$$

Formulas (32) show that the steady, rotating hurricane can exist even without horizontal travel. Here the heat supply $\tau_{0}$ to the adiabatic EW jet is entirely produced by the condensation heat only due to moisture vaporization.

\subsection{Numerical Illustrations}

\section{1) Accepted and calculated parameters}

Geometrical parameters known for the "standard" hurricane are: $r_{i}=20 \mathrm{~km}, \quad r_{e}=40 \mathrm{~km}, h_{e}=1.5 \mathrm{~km}$, and $r_{\text {ext }}=400 \mathrm{~km}$. Calculated geometrical parameters are: $q_{0}=3, \alpha=1 / 8, \varphi_{1}\left(q_{0}\right) \approx 1.25, \varphi_{2}\left(q_{0}\right) \approx 0.033$, $\kappa \approx 0.16, \quad \xi_{i}=0.5, \varepsilon=0.1$, and $\xi_{\text {ext }}=10$.

Physical parameters are- $T_{a}^{0}=300 \mathrm{~K}\left(27^{\circ} \mathrm{C}\right)$ with $\sqrt{R T_{a}^{0}}=295 \mathrm{~m} / \mathrm{s} ; c_{p}=10^{3} \mathrm{~J} \cdot \mathrm{K}^{-1} / \mathrm{kg}$ and $L=2.3 \times 10^{6} \mathrm{~J} / \mathrm{kg} \quad[28] ; C_{D} \approx 3 \times 10^{-3}, C_{E m} \approx 3 \times 10^{-5}, \quad v=1.8$, and $\lambda_{0}=11.7$.

Note that values $C_{D}$ and $C_{E m}$ being chosen here arbitrarily are still reasonable. Evidently, decreasing $C_{D}$ and increasing $C_{E m}$ will increase the severity of hurricane.

Parameters calculated from (29) are shown in Table 2.

2) Results of calculations. Using Table 2, the basic variables of hurricane for both cases were calculated and shown in Table 3 and Table 4. It was shown in [26] that the stability condition $\tau_{0}>\tau_{c}$ is satisfied here.

In both the cases, the most striking result of calculations is a high increase in temperature $\Delta T_{0}$ at the upper part of HBL EW, close to the observed values [2]. It clearly indicates the leading role of condensation. High increase in temperature $\Delta T_{+}$of warm air band only slightly contributed in $\Delta T_{0}$. Also, the vertical wind speed component $u_{0}$ is only slowly growing in both cases, tangential one $u_{\varphi m}$ is also growing, albeit not highly, but more in the affinity case. The radial wind speed component $\left|u_{r e}\right|$ slightly decreases with growing either $w_{s}$ or $\Delta T_{+}$. It means that due to the mass balance, the increasing rate of air entering the adiabatic EW jet from the HBL creates the lower value of initial tangent $\left|r_{e}^{\prime}\right|$ of the hurricane EW jet.

Table 2. Calculated non-dimensional parameters of standard hurricane.

\begin{tabular}{ccccccccc}
\hline Parameter & $\lambda$ & $X$ & $v$ & $\gamma_{1}$ & $\gamma_{2}$ & $\gamma_{3}$ \\
\hline Value & 0.0351 & 0.00341 & 1.64 & 0.727 & 0.00248 & 0.1 \\
\hline
\end{tabular}


Table 3. Results of calculations of basic variables in hurricane travel under given values of sailing wind $w_{s} \quad\left(\Delta T_{+}=0\right)$. The values of temperatures are in ${ }^{\circ} \mathrm{C}$; velocities in $\mathrm{m} / \mathrm{s}$.

\begin{tabular}{cccccccc}
\hline$w_{\mathrm{s}}, \mathrm{m} / \mathrm{s}$ & $u_{\rho m}, \mathrm{~m} / \mathrm{s}$ & $u_{0}, \mathrm{~m} / \mathrm{s}$ & $\left|u_{r e}\right|, \mathrm{m} / \mathrm{s}$ & $\tau_{-}$ & $\Delta T_{-}{ }^{\circ} \mathrm{C}$ & $\tau_{0}$ & $\Delta T_{0}{ }^{\circ} \mathrm{C}$ \\
\hline 0 & 49.5 & 1.74 & 20.0 & 0 & 0 & 0.097 & 8.31 \\
5 & 50.4 & 1.77 & 18.4 & 0 & 0 & 0.097 & 8.31 \\
10 & 51.3 & 1.80 & 16.8 & 0 & 0 & 0.097 & 8.31 \\
15 & 52.2 & 1.83 & 15.2 & 0 & 0 & 0.097 & 8.31 \\
\hline
\end{tabular}

Table 4. Results of calculations of basic variables in affine travel of hurricane under given values of $\Delta T_{+}{ }^{\circ} \mathrm{C}\left(w_{s}=0\right)$.The values of temperatures are in ${ }^{\circ} \mathrm{C}$; velocities in $\mathrm{m} / \mathrm{s}$.

\begin{tabular}{ccccccccccc}
\hline$\Delta T_{+}$ & $\tau_{+}$ & $u_{\varphi m}$ & $w_{a}$ & $u_{0}$ & $\left|u_{r e}\right|$ & $\tau_{-}$ & $\Delta T_{-}$ & $\tau_{0}$ & $\Delta T_{0}$ \\
\hline 0 & 0 & 49.5 & 0 & 1.74 & 17.4 & 0 & 0 & 0.097 & 8.31 \\
3 & 0.035 & 50.5 & 3.18 & 1.77 & 16.7 & 0.002 & 0.17 & 0.099 & 8.48 \\
6 & 0.070 & 52.6 & 6.62 & 1.85 & 16.4 & 0.008 & 0.69 & 0.105 & 9.00 \\
10 & 0.117 & 57.1 & 12 & 2.00 & 16.2 & 0.022 & 1.88 & 0.119 & 10.2 \\
\hline
\end{tabular}

Calculations of the radial distributions of surface pressure and wind for hurricane Frederic, 1979, using the data according to paper [31], were detailed in Ref. [26]. Comparison of data with our rough calculations is shown in Figure 13(a) and Figure 13(b).

\section{On the Hurricane Genesis and Maturing [27]}

The emergence of hurricanes is still mysterious. Many observations of initial stages of hurricanes (e.g. see the text [2]) found a threshold value of vorticity, exceeding which the hurricane is maturing. Analyses in papers by Ooyama [17] [18] and Emanuel [19] [20] have a mutual defect-adjustable diffusivity of angular momentum to fit the data. Also, most hurricanes in Atlantics are formed in near equator zone, indicating the importance of Coriolis factor, which was not considered in the above papers.

Paper [27] proposed a two-steps scenario of hurricane's genesis. In the first step, an emerged plume of warm and humid air formed in the near equator zone, moves upward (see the model of plume dynamics in [27]). In the second step, the plume captures the rotation from a horizontally sheared wind, with restructuring of the plume and acquiring an initial value of angular momentum. If this plume is stable, the maturing stage begins. In this case the hurricane grows in the radial direction, presumably caused by the K-H instability with radial propagation into ambient air under action of Earth rotation.

To describe the maturing stage of hurricanes we first consider the quasi-static relation for angular momentum extended to the external boundary $r_{a}(t)$ of hurricane:

$$
M(t)=[\omega(t)+\Omega] r_{a}^{2}(t) .
$$

The absolute $u_{\varphi q}(t)$ and relative $u_{\varphi}(t)$ tangential velocities are then defined as:

$$
u_{\varphi a}=M / r_{a}, \quad u_{\varphi}=M / r_{a}-\Omega r_{a} .
$$

The slow evolution of $r_{a}(t)$ and $M(t)$ is now described by two heuristic equations:

$$
\begin{aligned}
& \mathrm{d} r_{a} / \mathrm{d} t \approx k u_{\varphi}=k\left(M / r_{a}-\Omega r_{a}\right) \quad(k=\text { const } \sim 1) \\
& \mathrm{d} M / \mathrm{d} t \approx u_{\varphi a} \mathrm{~d} r_{a} / \mathrm{d} t \quad\left(u_{\varphi a} \approx \text { const }\right)
\end{aligned}
$$

The first equation in (35) describes propagation of the hurricane front due to the K-H instability with the relative rotational velocity at the boundary $r=r_{a}$. The second equation in (35) assumes that the radius change due 


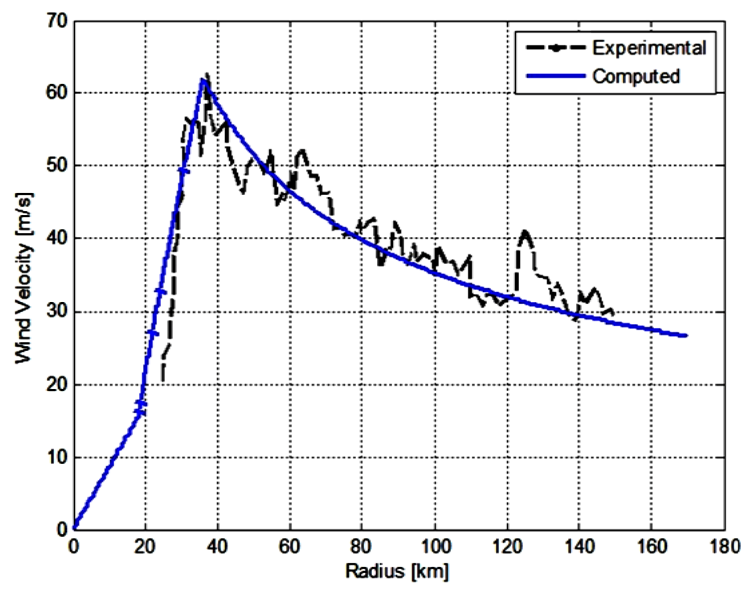

(a)

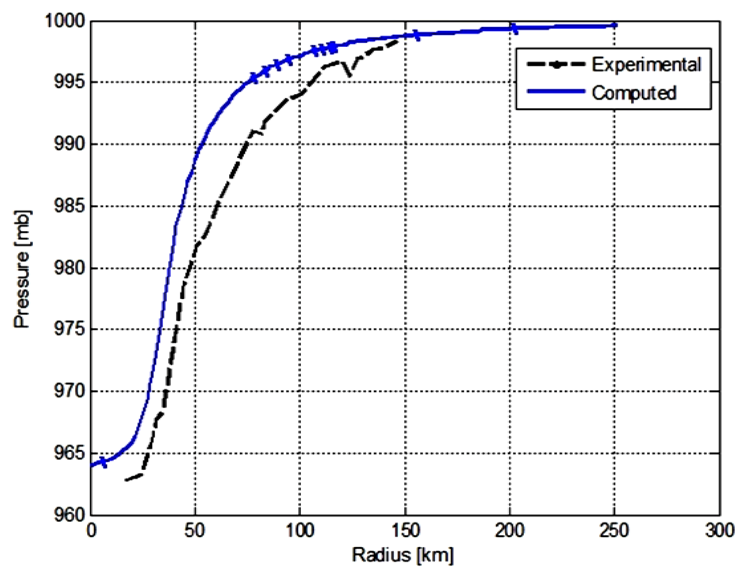

(b)

Figure 13. Comparison of our adiabatic calculations and measurements [31] for radial distributions of tangential wind and surface pressure for hurricane Frederic for measurements on 09/11/1979: (a) Tangential wind; (b) Surface pressure; maximal wind speed $u_{\varphi \max }=62 \mathrm{~m} / \mathrm{s}$, radius of eye jet $r_{e}=36 \mathrm{~km}$. Dashed and solid lines are measurements and calculations, respectively.

to the radial propagation of unstable boundary is the dominant contribution in the change of angular momentum.

The initial conditions are:

$$
r_{a}(0)=r_{0}, \quad M(0)=M_{0}=\left(\omega_{0}+\Omega\right) r_{0}^{2} \quad\left(\omega_{0} \approx \dot{\gamma}_{0}\right) .
$$

Here $\dot{\gamma}_{0}$ is the horizontal shear of wind initiated the plume rotation.

The solution of Equations (33)-(35) with conditions (36) is:

$$
r_{a}(t)=r_{0}\left[1+\left(\omega_{0} / \Omega\right)\left(1-\mathrm{e}^{-\Omega k t}\right)\right] ; \quad M(t)=\left(\omega_{0}+\Omega\right) r_{0}^{2}\left[1+\left(\omega_{0} / \Omega\right)\left(1-\mathrm{e}^{-\Omega k t}\right)\right]
$$

Formulas (37) show that depending on sign $\omega_{0}$, the plume can rotate in cyclonic or anti-cyclonic directions.

1) In the cyclonic case $\omega_{0}>0$, hurricane propagates outwards. It is the maturing case, when he functions, $r_{a}(t), M(t)$ and $\omega(t)$ monotonically grow to their stationary values,

$$
r_{s}=r_{0}\left(1+\omega_{0} / \Omega\right) ; \quad M_{s}=\Omega r_{0}^{2}\left(1+\omega_{0} / \Omega\right)^{2} ; \quad \omega_{s}=0 ; \quad u_{\varphi \max }=\omega_{0} r_{0}\left(2+\omega_{0} / \Omega\right) .
$$

2) In the anti-cyclonic case $\omega_{0}<0$, the disturbances propagate inwards, which cause the collapsing hurricane either in finite or in infinite time.

Thus the model selects as only stable, the cyclonic initial rotation, which naturally explains the observed cyclonic rotation of matured hurricanes. However, the model does not describe the observed threshold in value of $\omega_{0}$, seemingly because of the linear character of the first equation in (37).

To illustrate the model predictions we choose the following parameters $k \approx 1, \quad \Omega=5.5 \times 10^{-6} \mathrm{~s}^{-1}$, $\omega_{0}=10^{-4} \mathrm{~s}^{-1}, \quad r_{0}=25 \mathrm{~km}$. Calculations due to (37) and (38) yield:

i) Characteristic time of hurricane development: $t_{s} \approx 3 / \Omega \approx 5.45 \times 10^{5} \mathrm{sec} \approx 6.3$ days ;

ii) Characteristic radius of developed hurricane: $r_{s}=r_{0}\left(1+\omega_{0} / \Omega\right) \approx 479 \mathrm{~km}$;

iii) Maximum speed of developed hurricane: $u_{\varphi \max }=\omega_{0} r_{0}\left(2+\omega_{0} / \Omega\right) \approx 50.4 \mathrm{~m} / \mathrm{s}$;

iv) The grow of angular momentum: from $M_{0} \approx 6.59 \times 10^{4}$ to $M_{s} \approx 1.2 \times 10^{6} \mathrm{~m} / \mathrm{s}$.

These results are consistent with observations in text [2] that the initial tropical cyclone is transformed into a hurricane during 5 - 6 days after the action of wind with vorticity $\omega_{0} \approx 10^{-4} \mathrm{~s}^{-1}$.

\section{Conclusions}

The paper presents analytical two-layer hurricane model. The approach employed in the paper uses simplified aerodynamic equations for ideal humid gas with additional models for heat transfer, evaporation and condensation. It mostly avoids the common turbulent approximations, except a thin near-water sub-layer. 
Analysis of adiabatic aerodynamic modeling in the hurricane upper layer reveals a "hyperboloid" structure of eye wall (EW) jet. The radial and vertical distributions of basic variables have been theoretically calculated. It was found that upper layer of hurricane is stable when the thermal heat supplied into the layer exceeds the adiabatic cooling. The model also explains the change in the cyclonic/anti-cyclonic directions of hurricane rotation, as well as the directions of radial wind component in lower and upper parts of hurricane.

The model of hurricane boundary layer (HBL) employs aerodynamic approach only in its upper sub-layer and matches it with the turbulent approach in its lower sub-layer. The increase in the wind angular momentum in HBL is explained as an additional generation of wind by ocean waves propagating out of HBL EW. A dramatic effect of ocean spray and its radial distribution on evaporation has been modeled taking into account the ocean whitecaps generated by wind. A high increase in temperature in the upper sub-layer of HBL has been modeled by the condensation jump.

The balance relation applied to the HBL EW, presented the basic parameters governing the space distributions of field variables in hurricane via two external parameters-the sailing wind and horizontal temperature of a warm air band.

Additionally, a rude model for the hurricane genesis and maturing has also been developed. It explains the reason of cyclonic rotation of hurricanes.

All examples in the paper demonstrated a good correspondence with the existing observations when using common data for geometrical, fluid mechanical and thermodynamic parameters of hurricane.

It finally should be noted that developing the hurricane structure during hurricane genesis and maturing presents a very challenging numerical problem which by no means could be resolved by simplified analytical approaches.

The results of the paper could be used for easy tune-up of complicated numerical models, which take into account real interaction of hurricane with environment.

\section{Acknowledgements}

The author thanks Dr. A. Benilov for extensive and highly productive discussions, as well as the participants of Physical Science Division Seminar at NOAA in Boulder, CO (July, 2012). A lot of thanks are also given to former PhD Student, Dr. A. Gagov for help in calculations and graphics, and Dr. A. Voronovich for patiently reading the paper and making valuable suggestions.

\section{References}

[1] Dunn, G.E. (1951) Tropical Cyclones. In: Compendium of Meteorology, American Meteorological Society, Boston, 887-901.

[2] Anthes, R.A. (1982) Tropical Cyclones, Their Evolution, Structure and Effects. American Meteorological Society, Science Press, Ephrata, 208 p.

[3] Hsu, S.A. (1988) Coastal Meteorology. Academic Press, New York, 260 p.

[4] Cotton, W.R. and Anthes, R.A. (1989) Storms and Cloud Dynamics. Academic Press, San Diego, 883 p.

[5] Ogawa, A. (1993) Vortex Flow. CRS Press, Inc., Boca Raton.

[6] Emanuel, K.A. (2005) Divine Wind. Oxford University Press, New York, 285.

[7] Emanuel, K.A. (1983) An Air-Sea Interaction Theory for Tropical Cyclones. Part I. Journal of the Atmospheric Sciences, 42, 1062-1071. http://dx.doi.org/10.1175/1520-0469(1985)042<1062:FCITPO>2.0.CO;2

[8] Emanuel, K.A. (1995) The Behavior of Simple Hurricane Model Using a Convective Scheme Based on Subcloud Layer-Entropy Equilibrium. Journal of the Atmospheric Sciences, 52, 3959-3968. http://dx.doi.org/10.1175/1520-0469(1995)052<3960:TBOASH>2.0.CO;2

[9] Emanuel, K.A. (1999) Thermodynamic Control of Hurricane Intensity. Nature, 401, 665-669. http://dx.doi.org/10.1038/44326

[10] Holland, G.I. and Merrill, R.T. (1984) On the Dynamics of Tropical Cyclone Structural Changes. Quarterly Journal of the Royal Meteorological Society, 110, 723-745. http://dx.doi.org/10.1002/qj.49711046510

[11] Holland, G.J. (1997) The Maximal Potential Intensity of Tropical Cyclones. Journal of the Atmospheric Sciences, 54, 2519-2541. http://dx.doi.org/10.1175/1520-0469(1997)054<2519:TMPIOT>2.0.CO;2

[12] Bister, M. and Emanuel, K.A. (1998) Dissipative Heating and Hurricane Intensity. Meteorology and Atmospheric 
Physics, 65, 233-240. http://dx.doi.org/10.1007/BF01030791

[13] Montgomery, M.T., Vladimirov, V.A. and Denissenko, P.V. (2002) An Experimental Study on Hurricane Mesovortices. Journal of Fluid Mechanics, 471, 1-32. http://dx.doi.org/10.1017/S0022112002001647

[14] Lighthill, J. (1999) Ocean Spray and the Thermodynamics of Tropical Cyclones. Journal of Engineering Mathematics, 35, 11-42. http://dx.doi.org/10.1023/A:1004383430896

[15] Barenblatt, G.I., Chorin, A.J. and Prostokishin, V.M. (2005) A Note Concerning the Lighthill Sandwich Model of Tropical Cyclones. Proceedings of the National Academy of Sciences of the United States of America, 102, 1114811150. http://dx.doi.org/10.1073/pnas.0505209102

[16] Kagan, B.A. (1995) Ocean-Atmospheric Interaction and Climate Modeling. Cambridge University Press, New York, 377. http://dx.doi.org/10.1017/CBO9780511628931

[17] Ooyama, K.V. (1982) Conceptual Evolution of the Theory and Modeling of the Tropical Cyclone. Journal of the Meteorological Society of Japan, 60, 369-379.

[18] Ooyama, K.V. (1983) Numerical Simulations of Life Cycle of Tropical Cyclones. Journal of the Atmospheric Sciences, 26, 3-40. http://dx.doi.org/10.1175/1520-0469(1969)026<0003:NSOTLC $>2.0 . C O ; 2$

[19] Emanuel, K.A. (1989) The Finite Amplitude Nature of Tropical Cyclogenesis. Journal of the Atmospheric Sciences, 46, 3431-3456. http://dx.doi.org/10.1175/1520-0469(1989)046<3431:TFANOT>2.0.CO;2

[20] Emanuel, K.A. (1997) Some Aspects of Hurricane Inner-Core Dynamics and Energetic. Journal of the Atmospheric Sciences, 54, 1014-1026. http://dx.doi.org/10.1175/1520-0469(1997)054<1014:SAOHIC>2.0.CO;2

[21] Liu, Y., Zhang, D.-L. and Yao, M.K. (1997) A Multi-Scale Numerical Study of Hurricane Andrew, 1992. Part I: Explicit Simulation and Verification. Monthly Weather Report, 125, 3073-3093. http://dx.doi.org/10.1175/1520-0493(1997)125<3073:AMNSOH>2.0.CO;2

[22] Wang, Y. and Wu, C.-C. (2004) Current Understanding of Tropical Cyclone Structure and Intensity Changes-A Review. Meteorology and Atmospheric Physics, 87, 257-278. http://dx.doi.org/10.1007/s00703-003-0055-6

[23] Chan, C.L., Duan, Y. and Shay, L.K. (2001) Tropical Cyclone Intensity Change from a Simple Ocean-Atmosphere Coupled Model. Journal of the Atmospheric Sciences, 58, 154-172. http://dx.doi.org/10.1175/1520-0469(2001)058<0154:TCICFA>2.0.CO;2

[24] Leonov, A.I. (2008) Aerodynamic models for hurricanes I. Model description and horizontal motion of hurricane. http://arxiv.org/abs/0812.3173

[25] Leonov, A.I. (2008) Aerodynamic Models for Hurricanes II. Model of the Upper Hurricane Layer. http://Arxiv.Org/Abs/0812.3176

[26] Leonov, A.I. (2008) Aerodynamic Models for Hurricanes III. Modeling Hurricane Boundary Layer. http://arxiv.org/abs/0812.3178

[27] Leonov, A.I. (2008) Aerodynamic Models for Hurricanes IV. On the Hurricane Genesis and Maturing. http://arxiv.org/abs/0812.3180

[28] Curry, J.A. and Webster, P.J. (1999) Thermodynamics of Atmospheres and Oceans. Academic Press, London, 467.

[29] Yarin, A.L. (1993) Free Liquid Jets and Films: Hydrodynamics and Rheology. Longman Sci. \& Tech. with John Wiley \& Sons, Inc., New York, 446 p.

[30] Deppermann, C.E. (1947) Notes on the Origin and Structure of Philippine Typhoons. Bulletin of the American Meteorological Society, 28, 399-404.

[31] Powell, M. (1982) The Transition of Hurricane Frederic Boundary Layer Wind Field from the Open Gulf of Mexico to Landfall. Monthly Weather Review, 10, 1912-1932. http://dx.doi.org/10.1175/1520-0493(1982)110<1912:TTOTHF>2.0.CO;2

[32] Zhao, D. and Toba, Y. (2001) Dependence of Whitecup Coverage on Wind and Wind-Wave Properties. Journal of Oceanography, 57, 603-615. http://dx.doi.org/10.1023/A:1021215904955

[33] Hwang, P.A. and Sletten, M.A. (2008) Energy Dissipation and Wind-Generated Whitecup Coverage. Journal of Geophysical Research, 113, Article ID: CO2012. http://dx.doi.org/10.1029/2007JC004277

[34] Landau, L.D. and Lifshitz, E.M. (1986) Theoretical Physics. Vol. IV: Fluid Mechanics. Section 132 (in Russian), Nauka, Moscow, 733. 
Appendix: Structural Functions in the Section 3

$$
\begin{aligned}
& \Phi(\alpha, r, z)=q^{2} \alpha^{2}+(1-\alpha)^{2}(s-1)+x^{2}\left(1-s^{-1}\right)-2 x(1-\alpha) \ln s-\frac{\left(u_{r e}\right)^{2}(s-1)^{2}(q+1)}{2 \mu s} \\
& p_{0}(\alpha, z)=1-\frac{\mu}{1-z}\left[\alpha^{2}+\frac{1}{q+1}+\frac{(1-\alpha)^{2}}{q}+\frac{x^{2}}{q(q+1)}-\frac{2 x(1-\alpha)}{q^{2}} \ln (q+1)\right] \\
& x=1-\alpha-\alpha q ; \quad s=r^{2} ; \quad \alpha=0.5 /\left(q_{0}+1\right) \\
& F_{\alpha}(q)=\frac{3-5 \alpha^{2}+2 \alpha}{2}-2 \frac{(1-\alpha)^{2}}{q}+\frac{3(1-\alpha)^{2}-4 \alpha(1-\alpha) q+\alpha^{2} q^{2}}{q^{2}} \ln (q+1)
\end{aligned}
$$


Scientific Research Publishing (SCIRP) is one of the largest Open Access journal publishers. It is currently publishing more than 200 open access, online, peer-reviewed journals covering a wide range of academic disciplines. SCIRP serves the worldwide academic communities and contributes to the progress and application of science with its publication.

Other selected journals from SCIRP are listed as below. Submit your manuscript to us via either submit@scirp.org or Online Submission Portal.
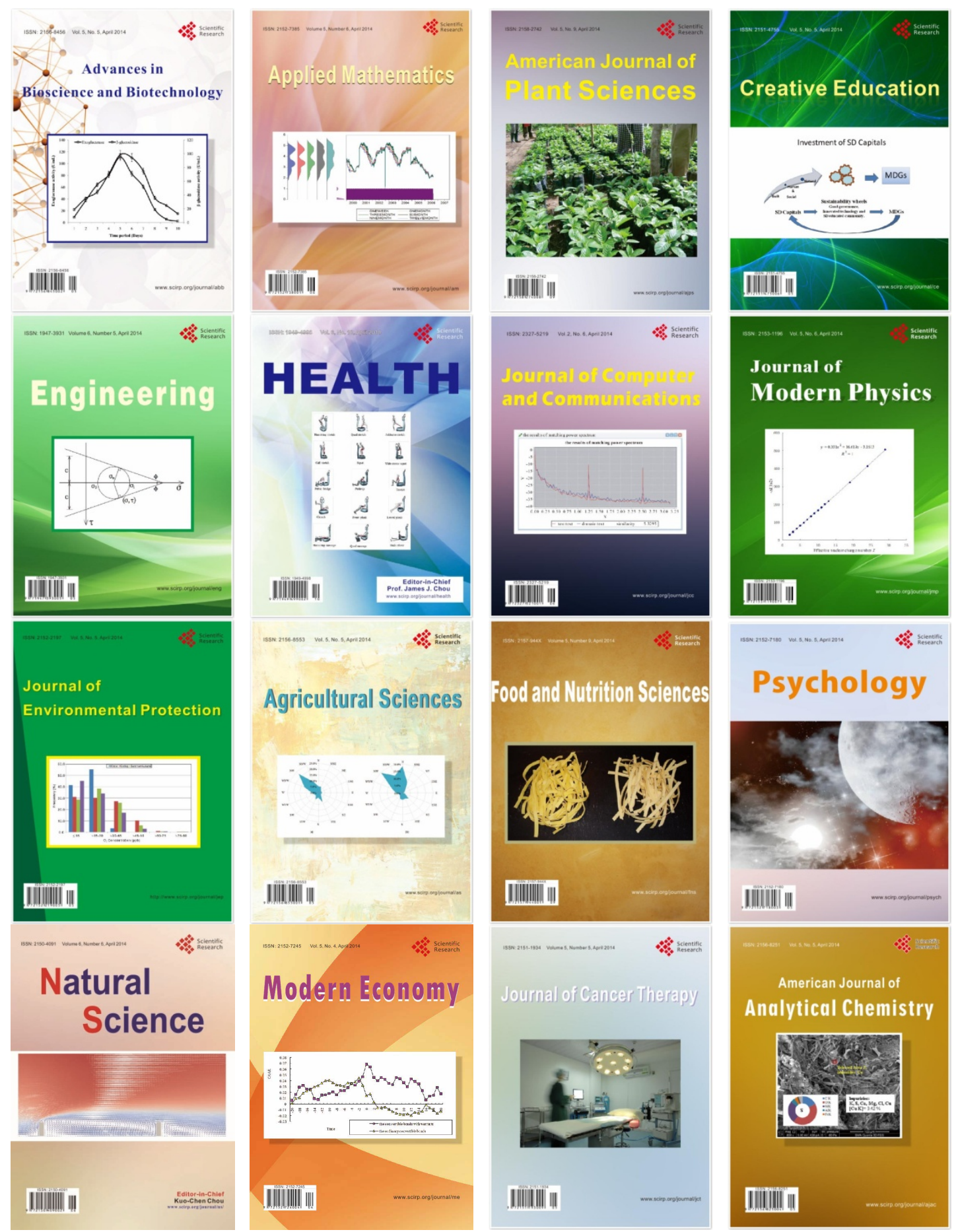